\title{
The Evolution and Arrest of a Turbulent Stratified Oceanic Bottom Boundary Layer over a Slope: Downslope Regime
}

\author{
XiAOZHOU RuAN AND ANDREw F. THOMPSON \\ Environmental Science and Engineering, California Institute of Technology, Pasadena, California \\ JOHN R. TAYLOR \\ Department of Applied Mathematics and Theoretical Physics, University of Cambridge, Cambridge, United Kingdom
}

(Manuscript received 19 April 2018, in final form 10 December 2018)

\begin{abstract}
The dynamics of a stratified oceanic bottom boundary layer (BBL) over an insulating, sloping surface depend critically on the intersection of density surfaces with the bottom. For an imposed along-slope flow, the cross-slope Ekman transport advects density surfaces and generates a near-bottom geostrophic thermal wind shear that opposes the background flow. A limiting case occurs when a momentum balance is achieved between the Coriolis force and a restoring buoyancy force in response to the displacement of stratified fluid over the slope: this is known as Ekman arrest. However, the turbulent characteristics that accompany this adjustment have received less attention. We present two estimates to characterize the state of the BBL based on the mixed layer thickness: $H_{a}$ and $H_{L}$. The former characterizes the steady Ekman arrested state, and the latter characterizes a relaminarized state. The derivation of $H_{L}$ makes use of a newly defined slope Obukhov length $L_{s}$ that characterizes the relative importance of shear production and cross-slope buoyancy advection. The value of $H_{a}$ can be combined with the temporally evolving depth of the mixed layer $H$ to form a nondimensional variable $H / H_{a}$ that provides a similarity prediction of the BBL evolution across different turbulent regimes. The length scale $L_{s}$ can also be used to obtain an expression for the wall stress when the BBL relaminarizes. We validate these relationships using output from a suite of three-dimensional large-eddy simulations. We conclude that the BBL reaches the relaminarized state before the steady Ekman arrested state. Calculating $H / H_{a}$ and $H / H_{L}$ from measurements will provide information on the stage of oceanic BBL development being observed. These diagnostics may also help to improve numerical parameterizations of stratified BBL dynamics over sloping topography.
\end{abstract}

\section{Introduction}

In the abyssal ocean, enhanced shear and turbulence occurs in a thin region near the seafloor known as the oceanic bottom boundary layer (BBL). The BBL is an important source of drag on mean ocean currents and eddies and plays a key role in global oceanic energy budgets (Wunsch and Ferrari 2004). However, significant disagreement exists in estimates of the global energy dissipation in the BBL. Previous studies have estimated that energy dissipated in the BBL can range from $0.2 \mathrm{TW}$ to as large as 0.83 TW (Wunsch and Ferrari 2004; Sen et al. 2008; Arbic et al. 2009; Wright et al. 2013), which can be compared with the 0.8-0.9 TW of energy input from the wind into the geostrophic circulation (Wunsch and Ferrari 2004; Scott and Xu 2009). In addition to sparse

\footnotetext{
Corresponding author: Xiaozhou Ruan,xruan@caltech.edu
}

observations, additional uncertainty in dissipation rates arises from a poor understanding of how stratification and bottom slopes combine to modify ocean flows over the seafloor.

Flow-topography interactions in the ocean may lead to the generation of meso-/submesoscale energetic turbulence (Gula et al. 2016) and internal gravity waves (Nikurashin and Ferrari 2011). The BBL can thus be a site of enhanced dissipation and water mass transformation (Armi 1978; Ruan et al. 2017). Contrary to classical arguments (e.g., Munk 1966), recent studies have suggested that BBLs over sloping topography are the primary locations for the upwelling of deep water needed to close the global overturning circulation (De Lavergne et al. 2016; Ferrari et al. 2016; De Lavergne et al. 2017). These arguments point to the BBL being the primary site of a convergent turbulent buoyancy flux needed to support diabatic upwelling. However, due to the relatively 
small spatial scale of the BBL and practical difficulties associated with deep-sea observations, accurate representation of the oceanic BBL in large-scale general circulation models (GCM) remains challenging.

Stratified BBLs over a flat bottom have been extensively studied in both nonrotating and rotating systems; the latter is known as the bottom Ekman layer (BEL). Direct numerical simulations (DNS) and large-eddy simulations (LES) have been carried out at different Reynolds numbers to study the structures of the BEL, Ekman transport, Ekman veering angle, and their dependence on the external stratification. As external stratification increases, turbulence is suppressed, and the BEL becomes thinner with a relatively unchanged depth-integrated transport (Coleman et al. 1990; Shingai and Kawamura 2002; Taylor and Sarkar 2008). The Ekman veering angle is reduced as compared with laminar theory, but the veering angle tends to increase with increasing external stratification in the lower part of the BEL (Taylor and Sarkar 2008; Deusebio et al. 2014).

A sloping bottom boundary introduces additional dynamics. In a stratified BBL, the insulating bottom boundary condition causes density surfaces, or isopycnals, to tilt downslope in the absence of an alongslope mean flow. In steady state, an upslope convective flux is induced to balance the vertical buoyancy diffusion, as shown by Phillips (1970) and Wunsch (1970). In a rotating system, the tilting isopycnals also induce an along-slope geostrophic flow due to the thermal wind relation. When rotation is combined with an imposed along-slope mean flow, the near-bottom crossslope Ekman transport is always smaller than in the flat-bottom case. This is due to the opposing buoyancy force in the cross-slope direction. Isopycnals tilt either up- or downslope, depending on the orientation of the along-slope mean flow; in this study, we only consider along-slope flows that induce downslope Ekman transport. If the buoyancy force is sufficiently large to balance the Coriolis force in the cross-slope direction, the system arrives at a steady state with negligible Ekman transport. This is the so-called Ekman arrest (MacCready and Rhines 1991), where the near-bottom velocity shear and thus the wall stress $\tau_{w}$ are also reduced, compared to flat-bottom cases. Here the wall stress is defined as

$$
\tau_{w}=\left.\rho_{0} \nu \frac{\partial u}{\partial z}\right|_{z=0}=\rho_{0} u_{*}^{2}
$$

where $\rho_{0}$ is a reference density, $\nu$ is the molecular viscosity, $u(z)$ is velocity parallel to the bottom, and $u *$ is the friction velocity. Critically, the steady Ekman arrested state has not been observed in the ocean, despite efforts aimed at closing the integrated momentum and buoyancy budget in the BBL (Trowbridge and Lentz 1998). Our results provide some insight into why observations of a steady Ekman arrest have been elusive.

Besides the steady state solutions introduced above, process studies have examined the time-dependent adjustment toward Ekman arrest. For studies that have not explicitly resolved turbulence in the BBL, typically one of two parameterizations is used. The first invokes a constant turbulent viscosity and diffusivity, which encapsulates the enhanced turbulent diffusion of momentum and buoyancy. Following early numerical studies by Weatherly and Martin (1978), MacCready and Rhines (1991) solved for an approximate Ekman arrest time scale $\tau_{\text {laminar }}$ for a laminar system and found $\tau_{\text {laminar }}$ depends on the slope Burger number $(\mathrm{Bu})$ :

$$
\tau_{\text {laminar }}=\frac{1}{S^{2} f \cos \alpha}\left(\frac{1 / \sigma+S}{1+S}\right) .
$$

Here, $S=\mathrm{Bu}^{2}=(N \sin \alpha / f \cos \alpha)^{2}$, where $N$ and $f$ are the buoyancy and Coriolis frequencies, respectively; $\alpha$ is the slope angle, and $\sigma$ is the turbulent Prandtl number. The scale $\tau_{\text {laminar }}$ represents the time required for the cross-slope Ekman transport to arrive at the negligible steady state value $M_{\text {Thorpe }}=\kappa_{\infty} \cot \alpha$, derived by Thorpe (1987). Here, $\kappa_{\infty}$ is the far-field diapycnal diffusivity, which is generally smaller than the BBL diffusivity, where vigorous mixing takes place. During Ekman arrest, the stratified BBL over a slope becomes thicker than the BEL thickness due to the diffusion of buoyancy into the interior. The analytical solutions in the case of constant viscosity and diffusivity pose a curious conclusion: the interior mean flow depends on background parameters, such as $N$ and $\alpha$. In other words, the interior velocity field is a part of the solution of the BBL system and cannot be viewed as a background forcing independent of BBL processes. By shaping the background mean flow, at least close to the ocean bottom, BBL dynamics may influence the interior circulation beyond classic Ekman spinup and spindown processes (Thomas and Rhines 2002; Benthuysen and Thomas 2013; Ruan and Thompson 2016).

As an alternative to a constant turbulent viscosity and diffusivity, various parameterizations have been applied as closures of turbulent momentum and buoyancy fluxes (e.g., the simple bulk Richardson number $R_{b}$-dependent and higher-order closure schemes). The latter includes the Mellor-Yamada schemes and the second-order closure implemented in a recent study examining the energy pathways in the Ekman arrest 
process (Umlauf et al. 2015). Trowbridge and Lentz (1991) have shown that a simple $R_{b}$-dependent parameterization is able to capture the general thickness evolution of the BBL, as compared to the Mellor-Yamada level-two turbulence closure used by Weatherly and Martin (1978). Brink and Lentz (2010, hereafter BL10) have tested different turbulent closure schemes and provided more accurate empirical expressions for the time scales associated with the Ekman arrest process. However, the turbulent characteristics associated with the BBL evolution have not been examined closely in the two approaches introduced above. This has motivated us to carry out LES simulations, which directly resolve the largest turbulent motions that were parameterized in BL10. We will show that the BBL reaches a relaminarized state in which turbulence is suppressed before evolving to the final arrested state.

Describing the Ekman arrest process as a function of time is useful; however, ocean observations often do not fit neatly into this "initial value" approach. Determining the BBL's time history, or the stage of the BBL's turbulent evolution as it approaches the arrested state, remains difficult. Here, we provide a framework that both classifies and identifies various BBL stages, spanning fully turbulent flat-bottom cases to Ekman arrested states, based on instantaneous bulk structures. A key motivation is that this framework will allow for more accurate parameterizations of $\mathrm{BBL}$ processes in GCMs. Our theoretical derivation, described in section 2, suggests that different BBL stages are associated with transitions in turbulent characteristics. Therefore, we use a suite of LES (section 3) to simulate a stratified oceanic BBL over a slope with a downwelling-favorable mean flow (Fig. 1) in order to explore these regime transitions and to validate the theoretical predictions (section 4). The mean momentum and buoyancy budgets are diagnosed in section 5; discussions and conclusions are provided in section 6 . The goals of this study are threefold: (i) to quantify the effects of topographic slope and stratification on the BBL turbulent characteristics, as well as the wall stress, BBL thickness, and Ekman transport; (ii) to describe the detailed structure of stratified BBL over a slope; and (iii) to propose a unified description of the evolution of stratified BBL over a slope throughout all stages toward full arrest.

\section{Theoretical predictions}

We begin by introducing two expressions for the height of the bottom mixed layer (BML) $-H_{a}$ and $H_{L}$, or the "arrest height" and "relaminarization height"which can be determined from external parameters.

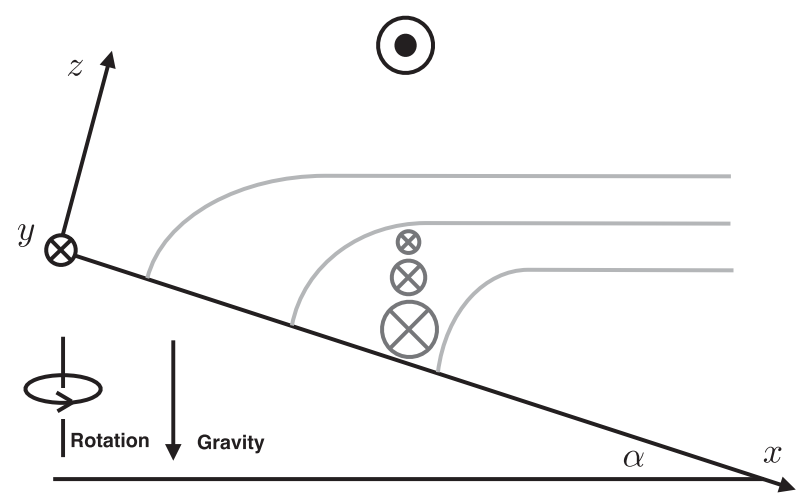

FIG. 1. Schematic of the BBL over a slope; gray curves indicate density surfaces. The coordinate is rotated by a slope angle $\alpha$. The barotropic mean flow is associated with a downslope Ekman transport. The thermal wind shear generated due to the tilting isopycnals is in the positive $y$ direction, opposite to the mean flow. The near-bottom velocity is the sum of the barotropic mean flow and the opposing thermal wind shear.

In this study, the BML refers to the region of weak vertical stratification, whereas the BBL describes the region with enhanced dissipation (e.g., a mixing layer). We first revisit a scaling for $H_{a}$ proposed by Trowbridge and Lentz (1991; section 2a). The second definition $H_{L}$ (section $2 \mathrm{~b}$ ) is, to our knowledge, new and based on Monin-Obukhov similarity theory. These values of the arrest height will prove to be critical not only for describing the arrested state, but also for classifying the approach to arrest, as shown in sections 4 and 5 .

\section{a. Momentum balance and arrest height}

As shown in Fig. 1, the coordinate system is rotated such that $x, y$, and $z$ denote the downslope, along-slope, and slope-normal directions, respectively, and $u, v$, and $w$ are the corresponding velocity components. To leading order, the boundary layer momentum equation in the cross-slope direction is given by

$$
\frac{\partial u}{\partial t}-f\left(v_{\text {total }}-\bar{v}\right)=-\alpha b-\frac{1}{\rho_{0}} \frac{\partial \tau^{x}}{\partial z}
$$

where $v_{\text {total }}$ and $\bar{v}$ (with magnitude $V_{\infty}$ ) are the total and far-field along-slope velocities, and $\tau^{x}$ is the total stress (molecular and Reynolds). Scalings for the near-seafloor Coriolis force (per unit mass) $F_{C}$ and buoyancy force (per unit mass) $F_{B}$ that balance during Ekman arrest are

$$
F_{C} \sim f V, F_{B} \sim \alpha b \sim \alpha^{2} N_{\infty}^{2} \Delta x \sim \alpha N_{\infty}^{2} H,
$$

where $V$ is the magnitude of the boundary layer alongslope velocity. The buoyancy force is proportional to the displacement of the stratification. For a uniform slope, 

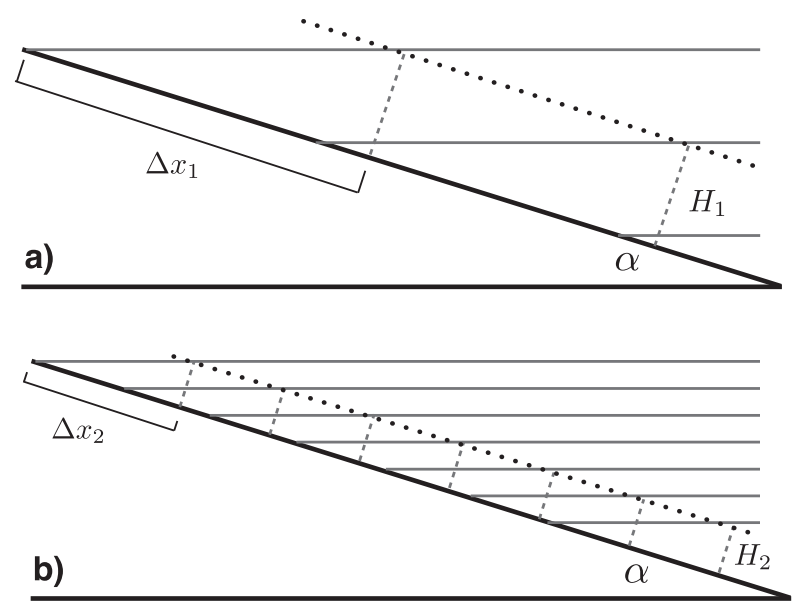

FIG. 2. Schematic of the arrest height $H_{a}$ in sloping BBLs with different stratifications. The dashed lines represent the isopycnals in the BML after they are advected downslope. The dotted lines denote the top of the BML. For the same slope angle $\alpha$ and mean flow magnitude $V_{\infty}$, fluid with stronger stratification $N_{\infty}^{2}$ requires a smaller $H_{a}$ to generate a buoyancy force to balance the Coriolis force $f V_{\infty}$ in the cross-slope direction. Here, $\Delta x_{1} N_{1}^{2}=\Delta x_{2} N_{2}^{2}$, so $H_{1} / H_{2}=N_{2}^{2} / N_{1}^{2}$. Terms $N^{2}$ and $H$ are the background stratification and arrest height associated with a weakly and a strongly stratified BBL (subscripts 1 and 2 , respectively).

this is approximated using the cross-slope isopycnal displacement length scale $\Delta x$ (Fig. 2), where $\Delta x \approx H / \alpha$, and $H$ is the height of the BML where stratification is smaller than $30 \%$ of the background stratification $N_{\infty}^{2}$. The extra slope angle $\alpha$ in the expression for $F_{B}$ in (4) denotes the projection of an upward-pointing buoyancy force onto the cross-slope direction. In the arrested state where the total near-bottom flow is weak, $F_{C}$ and $F_{B}$ balance and can be expressed as

$$
F_{C}^{\text {arrest }}=f V_{\infty}, \quad F_{B}^{\text {arrest }} \approx \alpha N_{\infty}^{2} H_{a} .
$$

This yields an expression for the arrest height $H_{a}$ :

$$
H_{a} \approx f V_{\infty} /\left(\alpha N_{\infty}^{2}\right) .
$$

The same expression was proposed by Trowbridge and Lentz (1991) by assuming that the thermal wind shear $v_{z}=-\alpha N_{\infty}^{2} / f$ brings the total flow magnitude from the far-field value $V_{\infty}$ to zero near the bottom. This indicates that increasing the slope angle and stratification and/or reducing the mean flow magnitude leads to a reduction in the cross-slope displacement of the stratified fluid required to achieve Ekman arrest, or equivalently, a reduction in $H_{a}$. Using $f=10^{-4} \mathrm{~s}^{-1}$ and typical abyssal oceanic parameters $V_{\infty}=0.05 \mathrm{~m} \mathrm{~s}^{-1}, N_{\infty}^{2}=10^{-6} \mathrm{~s}^{-2}$, and $\alpha=0.005, H_{a}$ must be roughly $1000 \mathrm{~m}$ to generate a sufficiently large buoyancy force to balance the Coriolis force. This large value may partially explain why Ekman arrest is rarely observed in the abyssal ocean. However, for typical values over the continental slope where the pycnocline intersects topography- $V_{\infty}=0.05 \mathrm{~m} \mathrm{~s}^{-1}$, $N_{\infty}^{2}=10^{-5} \mathrm{~s}^{-2}$, and $\alpha=0.01-$ an $H_{a} \approx 50 \mathrm{~m}$ may be sufficient to achieve Ekman arrest.

Predictions for $H_{a}$ vary by four orders of magnitude across typical oceanic parameters (Figs. 3a-c). The nonlinear dependence of $H_{a}$ on different parameters warrants careful examination of BBL structures in different regimes, which is the focus of section 4 .

\section{b. Turbulent characteristics and relaminarization height}

An alternative definition of an arrest height begins by assuming that a complete balance between buoyancy and Coriolis forces requires the suppression of turbulence and turbulent stress. The competition between shear production and buoyancy flux can be characterized by the Obukhov length scale, which is defined by

$$
L \equiv \frac{-u_{*}^{3}}{k B},
$$

where $k=0.41$ is the von Kármán constant, and $B$ is the surface buoyancy flux. For an unstable BBL, where the buoyancy flux is upward $(B>0)$, the Obukhov length scale $L$ is negative, and it characterizes the relative importance of surface stress and convection in the production of turbulence. For a stable BBL, where the buoyancy flux is downward $(B<0), L$ is positive, and it corresponds to the transition depth (height above bottom) at which the stabilizing influence of stratification begins to suppress turbulence.

In the absence of a buoyancy flux at the wall in the oceanic BBL, (7) can be revised by replacing $B$ with the depth-integrated cross-slope buoyancy advection, which results in a new length scale, here called the "slope Obukhov length":

$$
L_{s} \equiv \frac{u_{*}^{3}}{k U N_{\infty}^{2} \alpha}
$$

where $U=\int_{0}^{\infty} u d z$ is the depth-integrated cross-slope transport. We show, using LES simulations, that the ratio of $H$ to $L_{S}$ captures the transition of the BBL from unstable to stable states and finally to an Ekman arrested state (section 4e). The dependence of $L_{s}$ on $U$ can be removed by relating the steady state Ekman transport over a slope to the friction velocity (BL10):

$$
U=u_{*}^{2} / f\left(1+\mathrm{Bu}^{2}\right)
$$

such that 

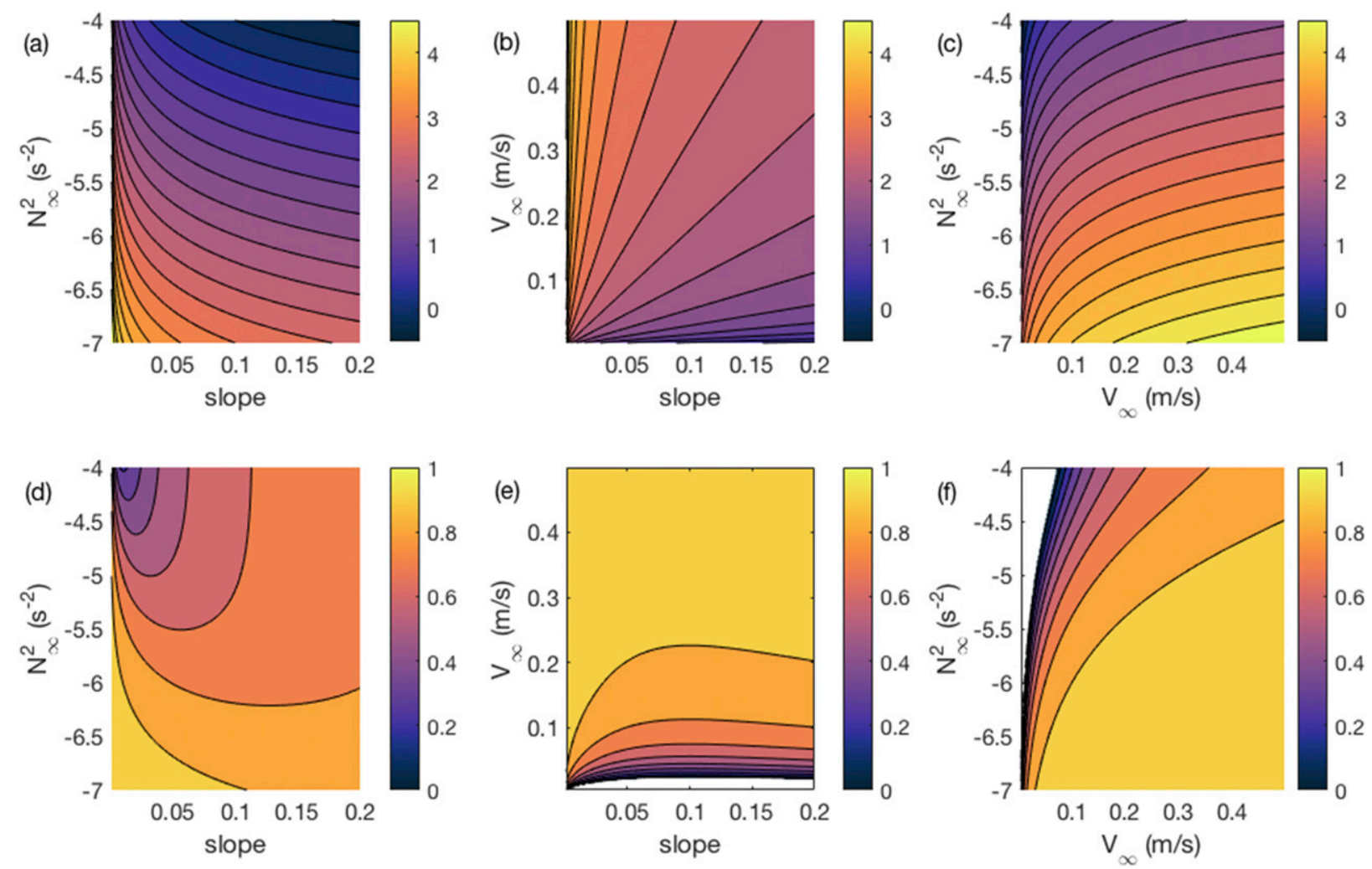

FIG. 3. (top) The predicted arrest height $H_{a}\left(\mathrm{~m}\right.$; logarithmic scale, e.g., $1=10 \mathrm{~m}$ for $H_{a}$ ) and (bottom) the ratio $H_{L} / H_{a}$ as a function of slope angle $\alpha$, background stratification $N_{\infty}^{2}$, and mean flow magnitude $V_{\infty}$. Estimates of $H_{a}$ and $H_{L}$ are based on the mean momentum balance [(6)] and turbulent characteristics [(14) with $\left.C_{d}=2 \times 10^{-3}\right]$, respectively. The parameters that are held fixed for different cases are (a),(d) $V_{\infty}=0.01 \mathrm{~m} \mathrm{~s}^{-1}$, (b),(e) $N_{\infty}^{2}=10^{-6} \mathrm{~s}^{-2}$, and (c),(f) $\alpha=0.01$.

$$
L_{s}=\left(1+\mathrm{Bu}^{2}\right) \frac{f u_{*}}{k \alpha N_{\infty}^{2}}
$$

It has been shown that the nondimensional viscous Obukhov length $L^{+}=L u * / \nu$ controls the turbulent state in stratified atmospheric boundary layers, such that for $L^{+}<100$, turbulence collapses and the boundary layer relaminarizes (Flores and Riley 2011). The Obukhov length $L$ characterizes the depth over which turbulence generation is unaffected by stratification, and $100 \nu / u *$ roughly denotes the upper limit of the viscous wall region (including the viscous sublayer, the buffer layer, and part of the lower log-law layer). Thus, $L<100 \nu / u *$ implies that turbulence suppression by stratification has penetrated into the viscous wall region, which results in turbulence collapse.

The physical interpretation of the slope Obukhov length $L_{s}$ is the same as the Obukhov length $L$. Assuming that turbulence in the oceanic BBL also collapses when the viscous slope Obukhov length

$$
L_{s}^{+}=L_{s} u_{*} / \nu=\left(1+\mathrm{Bu}^{2}\right) f u_{*}^{2} /\left(\nu k \alpha N_{\infty}^{2}\right)
$$

falls below a critical value $C$, the squared friction velocity associated with the transition from a turbulent to a relaminarized state is

$$
\left(u_{*}\right)^{2}=C \frac{\nu k \alpha N_{\infty}^{2}}{f\left(1+\mathrm{Bu}^{2}\right)} .
$$

When the friction velocity becomes smaller than the value predicted in (12), the BBL will transition to a laminar state. In section 4c, we show that the critical value for the constant $C$ in these simulations is also around 100 . Accounting for the reduction in the near-bottom, alongslope velocity due to the thermal wind shear, the revised expression for the wall stress using the quadratic law is

$$
\tau_{w}^{y} / \rho_{0}=C_{d} V_{b}^{2}=C_{d}\left(V_{\infty}-\alpha N_{\infty}^{2} H / f\right)^{2},
$$

where $C_{d}$ is the drag coefficient, and $V_{b}$ is the nearbottom flow magnitude. An expression for the relaminarization height is then given by

$$
H_{L}=\frac{f V_{\infty}}{\alpha N_{\infty}^{2}}-\left[\frac{C k \nu f}{\alpha N_{\infty}^{2} C_{d}\left(1+\mathrm{Bu}^{2}\right)}\right]^{1 / 2},
$$


a threshold for the BML thickness above which the BBL relaminarizes.

The scaling for $H_{a}$ in (6) is recovered when the second term in (14) is small (e.g., when the wall stress is negligible). When the BBL reaches the relaminarized state, the BML thickness $H_{L}$ is always smaller than the predicted $H_{a}$ for steady Ekman arrest. The scales $H_{a}$ and $H_{L}$ become more similar for small $\alpha$, weak $N^{2}$, and strong $V_{\infty}$ (Figs. 3d-f). Once the BBL is relaminarized, the only mechanism for further evolution to the final arrested state is via molecular diffusion. However, ubiquitous background perturbations are likely to make the relaminarized state difficult to sustain, providing another explanation for why a steady Ekman arrested state has not been observed.

In our LES simulations, we focus on BBL relaminarization, which we believe is of more oceanic relevance than the Ekman arrested state. We also note that both $H_{a}$ and $H_{L}$ are likely underestimated, compared with the true BML thickness, because of two assumptions. First, we assume that the tilted isopycnals can be represented by straight lines (Fig. 2). In reality, the isopycnals tilt smoothly toward the bottom, which yields a larger $H_{a}$ at steady state. Second, $V_{b}$ is defined at the bottom of the thermal layer rather than at $z=0$. Thus, we do not account for the thickness of the viscous layer in $H_{a}$, including the viscous sublayer, the buffer layer, and the lower part of the log layer.

\section{Numerical methods}

In the remainder of the paper, we show that the ratio of the mixed layer depth $H$ to $H_{L}$ is an important parameter for predicting relaminarization of the $\mathrm{BBL}$ over a slope, whereas the ratio of $H$ to $H_{a}$ describes the evolution of the BBL across a range of turbulent regimes toward complete arrest. The dependence of $H_{L}$ on small-scale turbulent properties of the BBL motivates the use of LES simulations, described below.

\section{a. Governing equations}

The LES-filtered Navier-Stokes equations under the Boussinesq approximation in a rotating frame can be written in dimensional form as

$$
\begin{gathered}
\frac{\partial u}{\partial t}+\mathbf{u} \cdot \nabla u-f v \cos \alpha=-\frac{1}{\rho_{0}} \frac{\partial p_{d}^{\prime}}{\partial x}-b \sin \alpha+\nu \nabla^{2} u \\
-\partial_{j} \tau_{1 j}^{d}, \\
\frac{\partial v}{\partial t}+\mathbf{u} \cdot \nabla v+f(u \cos \alpha-w \sin \alpha)=-\frac{1}{\rho_{0}} \frac{\partial p_{d}^{\prime}}{\partial y}+\nu \nabla^{2} v \\
-\partial_{j} \tau_{2 j}^{d},
\end{gathered}
$$

$$
\begin{aligned}
\frac{\partial w}{\partial t}+\mathbf{u} \cdot \nabla w+f v \sin \alpha= & -\frac{1}{\rho_{0}} \frac{\partial p_{d}^{\prime}}{\partial z}+b \cos \alpha \\
& +\nu \nabla^{2} w-\partial_{j} \tau_{3 j}^{d}
\end{aligned}
$$

$\frac{\partial b}{\partial t}+\mathbf{u} \cdot \nabla b-N_{\infty}^{2}(u \sin \alpha+w \cos \alpha)=\kappa \nabla^{2} b-\nabla \cdot \lambda^{d}, \quad$ and

$\nabla \cdot \mathbf{u}=0$

Here, $\nu$ and $\kappa$ are the molecular viscosity and diffusivity, respectively; $N_{\infty}^{2}=-\left(g / \rho_{0}\right) d \rho / d z$ is the background (nonevolving) stratification; $b=-g \rho^{\prime} / \rho_{0}$ is buoyancy, where $\rho^{\prime}$ is the density deviation from the background stratification; $p_{d}^{\prime}$ denotes the pressure deviation from the background hydrostatic balance, which has been removed from (17); and $\tau^{d}$ and $\lambda^{d}$ are the subgrid-scale (SGS) stress (with 1,2, and 3 representing the $x, y$, and $z$ directions) and buoyancy flux, respectively, which require SGS models for closure. The equations of motion are in a reference frame moving with the along-slope mean flow $\bar{v}$, with magnitude $V_{\infty}$. Therefore (16) gives the evolution of the perturbation velocity $v$ where $v=v_{\text {total }}-\bar{v}$, and $\bar{v}=-V_{\infty}$ for downslope Ekman transport conditions.

The dimensional variables are nondimensionalized using

$$
\begin{aligned}
(u, v, w) & =u_{*}\left(u^{\prime}, v^{\prime}, w^{\prime}\right), \quad(x, y, z)=\delta\left(x^{\prime}, y^{\prime}, z^{\prime}\right) \\
& =u_{*} / f\left(x^{\prime}, y^{\prime}, z^{\prime}\right), \\
p_{d}^{\prime}= & \rho_{0} u_{*}^{2} p^{\prime}, \quad b=N_{\infty}^{2} \delta b^{\prime}, \quad t=\delta / u_{*} t^{\prime} .
\end{aligned}
$$

The resulting nondimensional equations (with primes dropped, except for the pressure deviation) are

$$
\begin{aligned}
\frac{\partial u}{\partial t}+\mathbf{u} \cdot \nabla u-v \cos \alpha= & -\frac{\partial p^{\prime}}{\partial x}+\mathrm{Ri}_{*} b \sin \alpha \\
& +\operatorname{Re}_{*}^{-1} \nabla^{2} u-\partial_{j} \tau_{1 j},
\end{aligned}
$$

$$
\frac{\partial v}{\partial t}+\mathbf{u} \cdot \nabla v+(u \cos \alpha-w \sin \alpha)=-\frac{\partial p^{\prime}}{\partial y}+\operatorname{Re}_{*}^{-1} \nabla^{2} v
$$$$
-\partial_{j} \tau_{2 j}
$$

$$
\begin{aligned}
\frac{\partial w}{\partial t}+\mathbf{u} \cdot \nabla w+v \sin \alpha= & -\frac{\partial p^{\prime}}{\partial z}+\mathrm{Ri}_{*} b \cos \alpha \\
& +\operatorname{Re}_{*}^{-1} \nabla^{2} w-\partial_{j} \tau_{3 j}, \quad \text { and }
\end{aligned}
$$


TABLE 1. Summary of the simulation parameters. The slope Burger number $\mathrm{Bu}=N \sin \alpha / f \cos \alpha$, and other nondimensional parameters $\mathrm{Re}_{*}, \mathrm{Ri}_{*}$, and $\operatorname{Pr}$ are defined in (27). The values for $E_{a}$ and $E_{L}$ are given for the end of each experiment $t_{\text {end }} f$.

\begin{tabular}{cccccccccc}
\hline \hline Expt. & $\alpha$ & $\log _{10} N_{\infty}^{2}\left(\mathrm{~s}^{-2}\right)$ & $V_{\infty}\left(\mathrm{m} \mathrm{s}^{-1}\right)$ & $\mathrm{Bu}$ & $\mathrm{Re}_{*}$ & $\mathrm{Ri}_{*}$ & $\operatorname{Pr}$ & $E_{a}$ & $E_{L}$ \\
\hline A & 0.005 & -7 & 0.1 & 0.016 & 4232 & 10 & 5 & 0.002 & 0.002 \\
$\mathrm{~B}$ & 0.01 & -6.5 & 0.1 & 0.056 & 4232 & 31.6 & 5 & 0.014 & 0.015 \\
$\mathrm{C}$ & 0.01 & -6 & 0.1 & 0.1 & 4232 & 100 & 5 & 0.041 & 0.046 \\
$\mathrm{D}$ & 0.01 & -5.5 & 0.1 & 0.178 & 4232 & 316 & 5 & 0.130 & 0.157 \\
$\mathrm{E}$ & 0.01 & -5 & 0.1 & 0.316 & 4232 & 1000 & 5 & 0.349 & 0.492 \\
$\mathrm{~F}$ & 0.02 & -5 & 0.1 & 0.632 & 4232 & 1000 & 5 & 0.772 & 1.215 \\
$\mathrm{G}$ & 0.01 & -6 & 0.05 & 0.1 & 1352 & 100 & 5 & 0.058 & 0.070 \\
$\mathrm{H}$ & 0.01 & -5 & 0.05 & 0.316 & 1352 & 1000 & 5 & 0.503 & 1.060 \\
\hline
\end{tabular}

$$
\nabla \cdot \mathbf{u}=0
$$

Three nondimensional parameters govern the system: the friction Reynolds number $\left(\mathrm{Re}_{*}\right)$, friction Richardson number $\left(\mathrm{Ri}_{*}\right)$, and Prandtl number $(\mathrm{Pr})$, where

$$
\mathrm{Re}_{*}=\frac{u_{*} \delta}{\nu}=\frac{u_{*}^{2}}{f \nu}, \quad \mathrm{Ri}_{*}=\frac{N_{\infty}^{2} \delta^{2}}{u_{*}^{2}}=\frac{N_{\infty}^{2}}{f^{2}}, \quad \operatorname{Pr}=\frac{\nu}{\kappa} .
$$

Relevant nondimensional parameters used in the experiments are listed in Table 1. The parameters are chosen to explore their controls on the Ekman arrest process, ranging from a near-flat-bottom and unstratified limit to an experiment with the fastest arrest allowed in the model. The friction velocity $u *$ that appears in the nondimensional parameters does not include the effects of stratification (i.e., $u *$ is the friction velocity before stratification is introduced; see discussion in section $3 \mathrm{~b}$ ). The equations are solved subject to no-slip and insulating boundary conditions:

$$
\begin{aligned}
v & =V_{\infty}, \quad z=0, \\
u & =w=0, \quad z=0, \quad \text { and } \\
\frac{\partial b}{\partial z}+N_{\infty}^{2} & =0, \quad z=0 .
\end{aligned}
$$

The far-field boundary conditions are free slip and insulating for the momentum and buoyancy equations. Again, the bottom boundary condition is set to ensure $v_{\text {total }}=0$. Throughout, the small angle approximation ( $\sin \alpha \approx \alpha$ and $\cos \alpha \approx 1)$ is applied.

\section{b. Numerical details}

The simulations are performed using the computational fluid dynamics solver, DIABLO. Details of the numerical method can be found in Taylor (2008) and Bewley (2008). The background cross-slope density gradient remains constant $\left(M_{\infty}^{2}=-\alpha N_{\infty}^{2}\right)$ throughout the adjustment, determined by the sloping topography cutting through the vertically stratified fluid; there is no along-slope density gradient. The model solves for density perturbations to the background stratification. Thus, periodic boundary conditions are used in the $x$ and $y$ directions with uniform grid spacing, and the derivatives in these two directions are computed with a pseudospectral method (dealiased using the 2/3 rule). Staggered and stretched grids are used in the slopenormal direction with finer grid spacing close to the upper and lower boundaries. Derivatives in the slopenormal direction are treated with second-order finite differences. The time-stepping algorithm uses a mixed third-order Runge-Kutta/Crank-Nicolson method.

To examine the impact of finite stratification on the dynamics close to the wall, the LES experiments performed here are run with near-wall resolution (LESNWR), also called a resolved LES, which resolves at least $80 \%$ of the energy in the flow (Pope 2001; Sagaut 2006). Near the wall, turbulent motions scale with the viscous length $\delta_{\nu}=\nu / u *$, which places strong constraints on the model resolution. We placed the first two grid points in the viscous layer $z^{+}<5$, and the minimum resolution in the slope-normal direction is $\Delta_{z}^{+}=2$; in dimensional units, $\Delta_{z}=2 \nu / u *$. The uniform grid spacing in the slope-parallel directions are $\Delta_{x}^{+}=\Delta_{y}^{+} \sim 20$. The domain size is $30 \mathrm{~m}\left(L_{x}\right) \times 30 \mathrm{~m}\left(L_{y}\right) \times 60 \mathrm{~m}\left(L_{z}\right)$, respectively. A sponge layer of thickness $10 \mathrm{~m}$ is placed at the top of the domain to avoid reflection of internal gravity waves generated from the interaction of BBL turbulence with the pycnocline.

The background stratification can suppress the initialization of a turbulent BEL. To focus on the turbulent state, as opposed to the transition to a turbulent state, the simulations are spun up in multiple stages. First, an unstratified simulation is conducted with linear damping added to the momentum equations in the $x$ and $y$ directions until the system reaches quasi equilibrium; the uniform damping rate is half of the inertial frequency $f$. This stabilizes the flow and reduces inertial oscillations. The linear damping is then removed, allowing the flow to adjust to the background environment. Finally, a stable background stratification is incorporated into the 
simulation with a thin BML $(2-3 \mathrm{~m})$ near the bottom to ensure the viscous sublayer is unaffected by the stratification at the start (see an example initial stratification profile for $N_{\infty}^{2}=10^{-5} \mathrm{~s}^{-2}$ in Fig. 4). The strongest stratification used in these experiments is $N_{\infty}^{2}=10^{-5} \mathrm{~s}^{-2}$.

The LES-filtered governing equations are essentially a low-pass-filtered version of the Navier-Stokes equations with the resolved velocity field used to determine the SGS stress tensor $\tau_{i, j}^{\text {SGS }}$. Similar to the SGS model used by Taylor and Ferrari (2010), a constant Smagorinsky model was used in the simulations:

$$
\tau_{i, j}^{\mathrm{SGS}}=-2 C^{2} \bar{\Delta}^{2}|\bar{S}| \bar{S}_{i, j}
$$

Here, $C=0.13$ is the Smagorinsky coefficient, $\bar{\Delta}=$ $\left(\Delta_{x} \Delta_{y} \Delta_{z}\right)^{1 / 3}$ is the implicit LES filter width, and $S_{i, j}$ is the rate of strain tensor. The overbar denotes the filtered (or resolved) field. The SGS eddy viscosity from the Smagorinsky model is calculated as $\nu_{\mathrm{SGS}}=C^{2} \bar{\Delta}^{2}|\bar{S}|$ with the constant molecular viscosity explicitly used in the resolved field. A constant SGS Prandtl number $\operatorname{Pr}_{\mathrm{SGS}}=\nu_{\mathrm{SGS}} / \kappa_{\mathrm{SGS}}=1$ is used to calculate the SGS eddy diffusivity.

\section{Identification of turbulent regimes from large-eddy simulations}

A series of experiments were conducted to examine how topographic slope $\alpha$, stratification $N_{\infty}$, and background flow $V_{\infty}$ impact the evolution and bulk structures of the BBL. Table 1 provides the slope Burger number $(\mathrm{Bu})$, initial friction Reynolds number $\left(\mathrm{Re}_{*}\right)$ and friction Richardson number ( $\mathrm{Ri} *$ ), and Prandtl number (Pr). The ratios $H / H_{a}$ and $H / H_{L}$, at the end of each simulation, are also given. These experiments span a range of turbulent states, including some that are far from relaminarization.

Given sufficient time and water column depth, the adjustment of a stratified fluid over sloping topography is always toward the steady Ekman arrested state; the time to reach this state depends on external parameters. For experiments across a wide range of conditions, the nondimensional parameters $E_{a}=H / H_{a}$ and $E_{L}=H / H_{L}$, which represent the extent to which the BBL has approached the arrested and relaminarized states, can be used to classify different BBL dynamical regimes. Indeed, $E_{a}$ is equivalent to the ratio between the buoyancy and Coriolis force:

$$
E_{a}=H / H_{a}=\alpha N_{\infty} / f \times N_{\infty} H / V_{\infty}=\mathrm{Bu} / \mathrm{Fr} \approx F_{B} / F_{C},
$$

where $\operatorname{Fr}=V_{\infty} /\left(N_{\infty} H\right)$ is the Froude number. Thus, the magnitude of $E_{a}$ serves as a measure of the extent

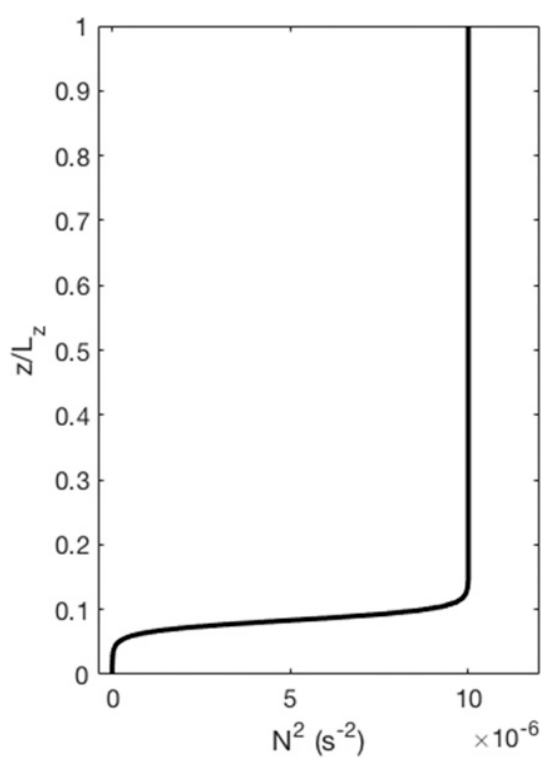

FIG. 4. An example initial stratification profile for $N_{\infty}^{2}=10^{-5} \mathrm{~s}^{-2}$; $L_{z}=60 \mathrm{~m}$ is the height of the domain. A thin mixed layer $(\sim 2 \mathrm{~m})$ is constructed to avoid the direct impact of stable stratification on the transition to turbulence in the BBL.

toward Ekman arrest (e.g., when $E_{a} \ll 1$, the BBL is far from the arrested state). Since the slope Burger number $\mathrm{Bu}$ in the ocean rarely exceeds unity, (32) implies that supercritical flows $(\mathrm{Fr}>1)$ are almost always far from arrest. Similarly, we can define

$$
E_{L}=H / H_{L},
$$

where $H_{L}$ is defined based on the critical viscous slope Obukhov length. Thus, $E_{L}=1$ and $L_{s}^{+}=100$ will be used interchangeably later to indicate a relaminarized state. Below, we discuss four sequential stages as the BBL evolves toward the steady arrested state: (i) weakly buoyant regime $\left(E_{a} \approx 0\right.$ and $\left.E_{L} \approx 0\right)$, (ii) buoyant regime $\left(0<E_{a}<1\right.$ and $\left.0<E_{L}<1\right)$, (iii) relaminarized regime $\left(0<E_{a}<1\right.$ and $\left.E_{L}=1\right)$, and (iv) Ekman arrested regime $\left(E_{a}=1\right.$ and $\left.E_{L}>1\right)$. A summary of the different regimes can be found in Fig. 5.

To highlight differences among these stages, we focus on the following properties: vertical stratification, vertical velocity profiles within the BBL, cross-slope transport, and friction velocity used to determine the wall stress. We discuss the connection among the newly proposed nondimensional parameters and turbulent characteristics in the BBL through the classic MoninObukhov similarity theory in section $4 \mathrm{e}$.

During all of these experiments, $H$ is continuously changing with time. The growth rates of the BML are well described by power law relationships $H \sim t^{b}$, 


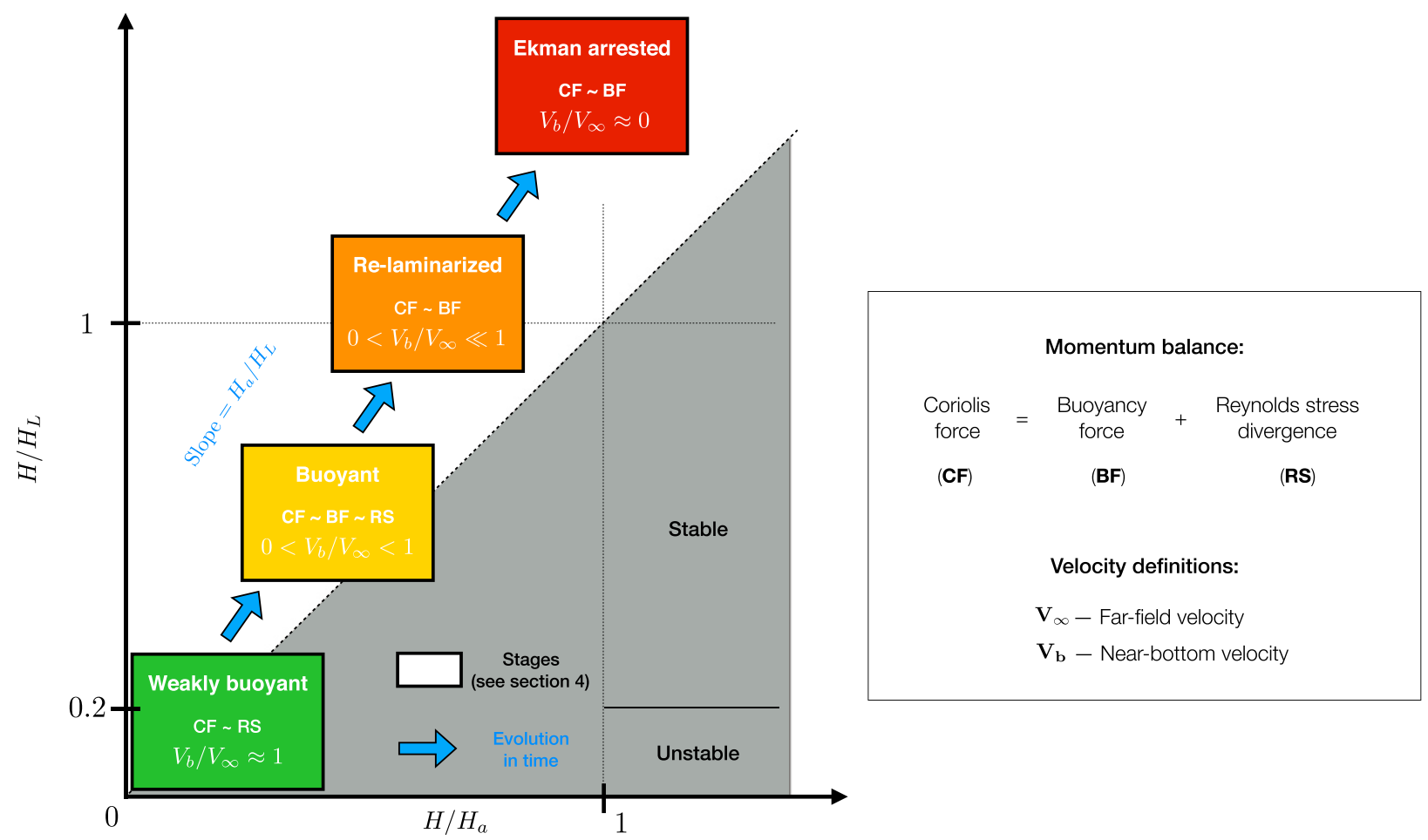

FIG. 5. Schematic representing the stages (boxes) in the approach to Ekman arrest; see discussion in section 4. The axes are the nondimensional numbers $E_{a}=H / H_{a}$ and $E_{L}=H / H_{L}$, defined in section 4. Each box summarizes the leading-order terms in the momentum balance and the ratio of far-field to near-bottom velocities, following the legend to the right.

although the exponent $b$ varies between different simulations (Fig. 6). The exponents fall between two limits. For the smallest initial $\mathrm{Bu}$, the convection is weak, and BML growth follows a $2 / 9$ power law, consistent with stress-driven mixed layer growth (Manucharyan and Caulfield 2015). For larger values of $\mathrm{Bu}, \mathrm{BML}$ growth follows a $1 / 2$ power law, consistent with a classic upright convection-driven mixed layer development (Deardorff et al. 1969). For the large $\mathrm{Bu}$ experiments, the sloping topography allows for larger downslope advection of buoyant fluid under heavier fluid that leads to the transition to stronger convective mixing. The simulated BML thickness is, overall, comparable to those in models that have used one-dimensional turbulence closure techniques. However, one-dimensional turbulence closure models largely account for turbulence production due to gravitational or Kelvin-Helmholtz instabilities in the bulk BBL and do not represent shear production at the wall (in the viscous sublayer). Additional analysis is needed to evaluate one-dimensional turbulence closures in simulating the Ekman arrest process.

Finally, to diagnose the vertical structure of velocity and other variables in the LES, a time average is applied over one near-inertial period to remove the effect of near-inertial oscillations. The centers of the averaging windows are labeled in Fig. 8 and indicated in Fig. 9 by the vertical dashed lines; the same average is applied in the figures shown below unless otherwise noted.

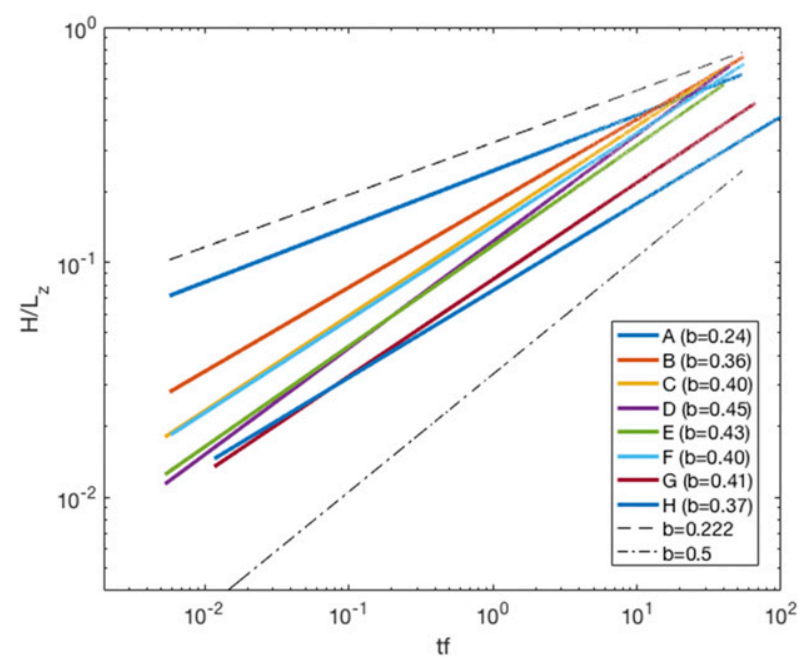

FIG. 6. The growth of the BML with time: $H / L_{z} \sim(t f)^{b}$. Different colors represent different simulations given in Table 1 . The dashed and dashed-dotted lines represent the reference power laws of the stress $(b=2 / 9)$ and upright convection-driven $(b=1 / 2) \mathrm{BBL}$ growth rates, respectively. 

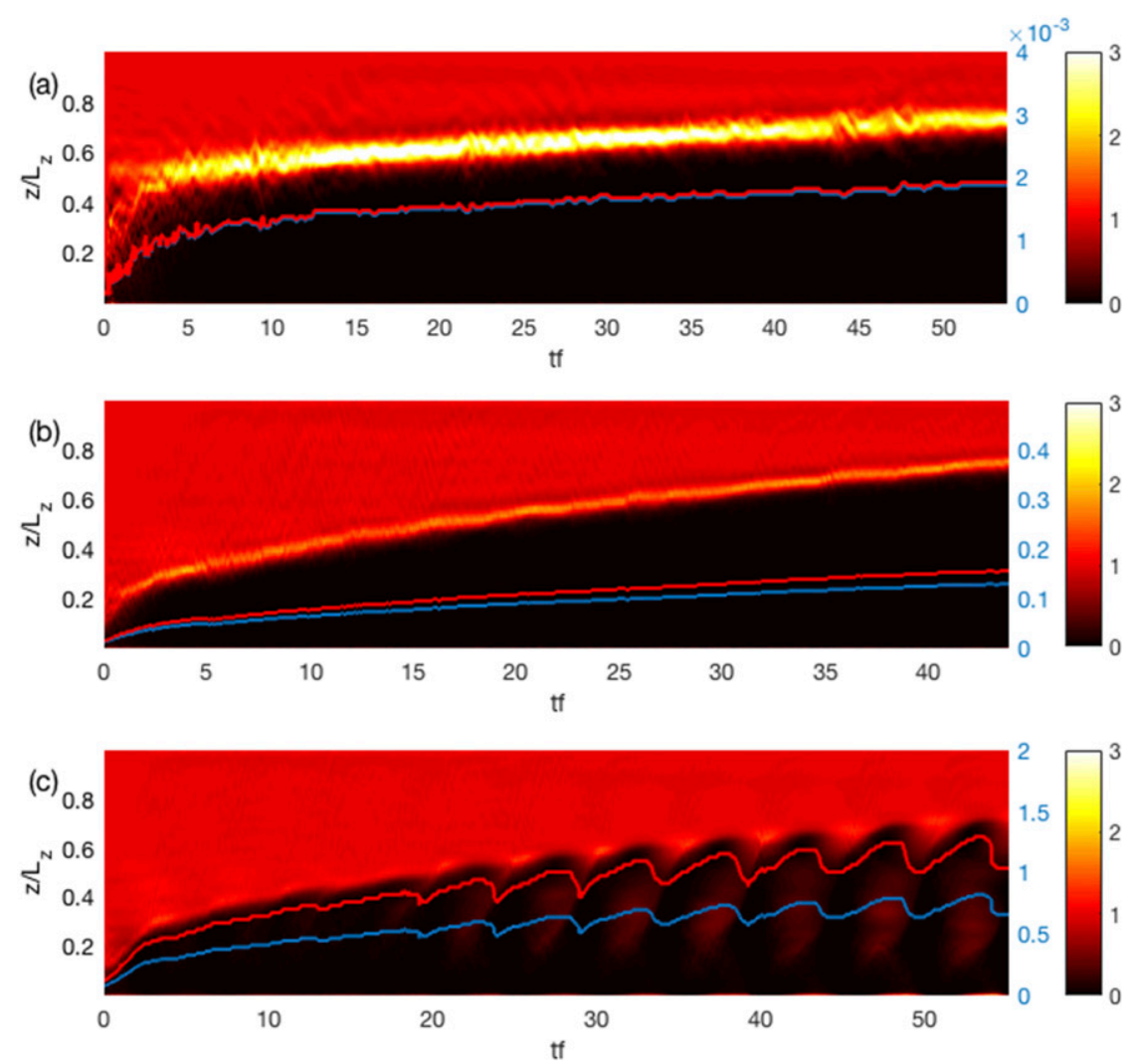

FIG. 7. Temporal evolution of the plane-averaged stratification $N^{2} / N_{\infty}^{2}$ in experiments (a) A, (b) $\mathrm{D}$, and (c) F, corresponding to initial values of $\mathrm{Bu}$ of $0.016,0.178$, and 0.632 , respectively. The evolution of the nondimensional parameters $E_{a}$ and $E_{L}$ are given by the blue and red curves, with the corresponding axis on the right in blue.

\section{a. Weakly buoyant regime: $E_{a} \approx 0$ and $E_{L} \approx 0$}

When the thickness of the BBL is small (i.e., $E_{a} \approx 0$ and $E_{L} \approx 0$ ), the dynamics of the BBL are similar to those described in studies of stratified BBL over a flat bottom (Taylor and Sarkar 2008; Deusebio et al. 2014). In this regime, the buoyancy force $F_{B}$ is weak in the cross-slope momentum balance [(3)]. Experiments with a gentle slope, a weak stratification, or a large mean flow all have large values of $H_{a}$ and $H_{L}$, and our LES experiments remain in the $E_{a} \approx 0$ and $E_{L} \approx 0$ regime throughout their duration (Table 1). Note, though, that all simulations pass through this stage at early times since $H \approx 0$ when the simulations are initialized.

In this stage, a strongly stratified pycnocline caps the BML. For instance, in experiment A, the stratification in the pycnocline is 3 times larger than the background value (Fig. 7a). Furthermore, the vertical structure of the horizontal velocity and veering angle through the BBL agree with flat-bottom Ekman layer dynamics (Figs. 8, 9a). After an initial adjustment, the cross-slope transport and friction velocity are relatively steady over the course of the simulations (Figs. 9b, 10a); both $U$ and $u *$ decrease as $E_{a}$ increases (Figs. 10b, 11).

\section{b. Buoyant regime: $0<E_{a}<1$ and $0<E_{L}<1$}

As $H$ grows, the importance of the buoyancy force $F_{B}$ in the cross-slope momentum equation begins to modify the characteristics of the BBL. In experiments with larger (initial) values of $\mathrm{Bu}$, the stratification in the pycnocline at the top of the BML is weaker (Fig. 7b) during this stage. This occurs because a more steeply sloping bottom or a stronger stratification causes buoyancy transfer to transition from being in the vertical direction to being primarily in the cross-slope direction. This weakens the tendency to form a pycnocline (see also the buoyancy budget in section $5 b$ ). This behavior may partially explain why the top of the BML in the ocean is not typically associated with a strong pycnocline (Armi 1978; Ruan et al. 2017).

As $E_{a}$ and $E_{L}$ become larger than 0.1, the cross-slope velocity profile penetrates deeper into the water column 

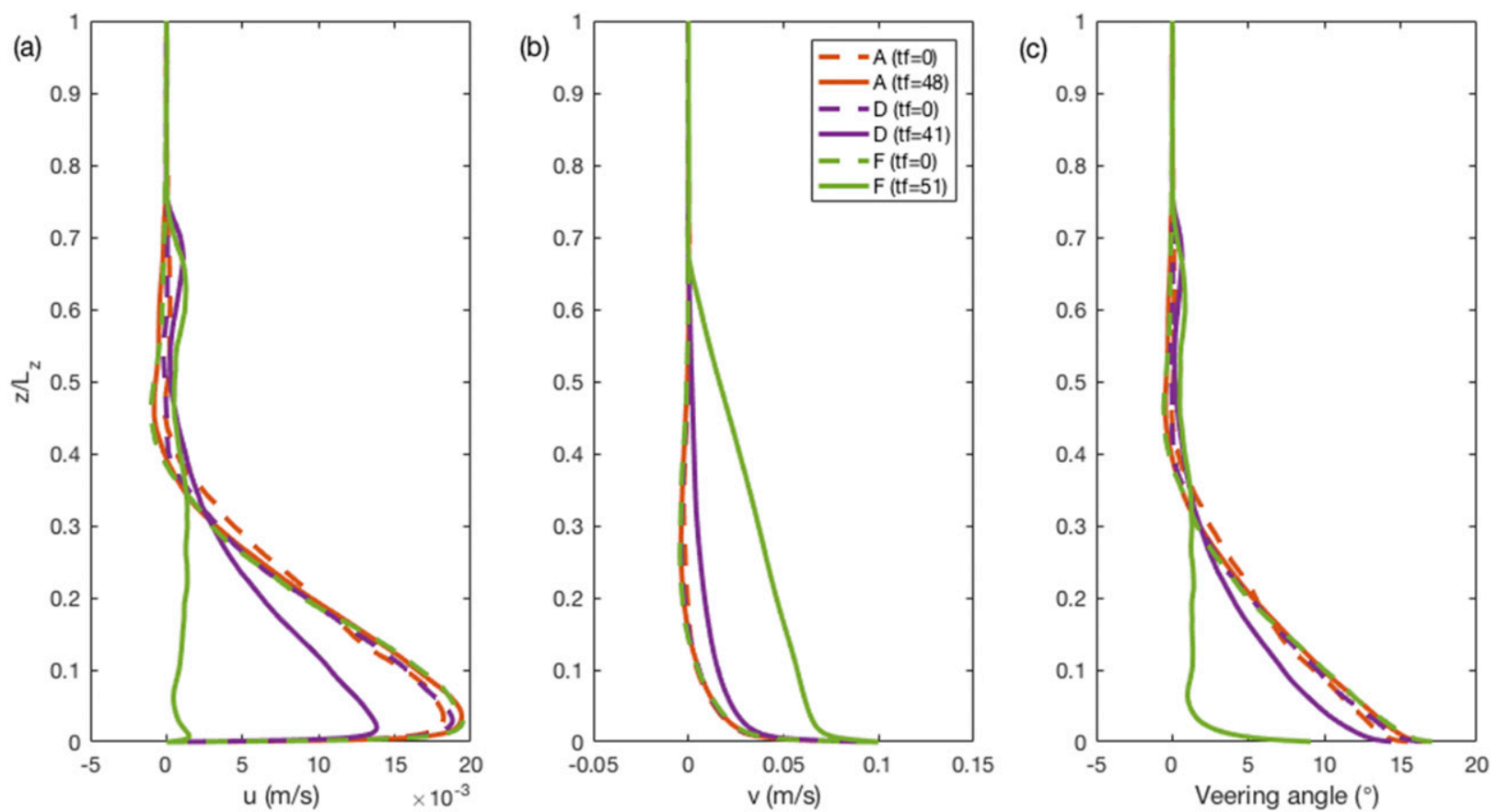

FIG. 8. The plane-averaged (a) cross-slope velocity, (b) along-slope velocity, and (c) Ekman veering angle at the beginning (dashed) and late stages (solid) of experiments $\mathrm{A}, \mathrm{D}$, and $\mathrm{F}$. The centers of the averaging windows are provided in (b) and correspond to the vertical dotted lines in Figs. 9a, 9c, and 9e. The along-slope velocity satisfies the no-slip boundary condition with the addition of $\bar{v}=-V_{\infty}=-0.1 \mathrm{~m} \mathrm{~s}^{-1}$.

(Figs. 8a, 9c), the cross-slope transport decays (Fig. 9d), and the friction velocity decreases (Fig. 10a), all as compared to the weakly buoyant regime (section 4a). In this regime, the deflection of isopycnals in the Ekman layer generates a thermal wind shear that opposes the along-slope velocity (Fig. 8b). This in turn reduces the velocity shear at the bottom, which leads to a smaller wall stress and friction velocity. Finally, the veering angle near the bottom decreases in response to the reduced wall stress, resulting in a smaller degree of turning of the along-slope flow, consistent with a weaker Ekman transport (Fig. 8c). While the veering angle is reduced, the thickness of the "veering layer" increases. This occurs because the thermal wind shear penetrates deeper than the Ekman layer. The Coriolis force $F_{C}$ then deflects the along-slope momentum into the cross-slope direction. This penetration of along-slope momentum is not entirely due to turbulent diffusion, but involves the buildup of the thermal wind shear-this is the "slow diffusion" process discussed by MacCready and Rhines (1991).

For all simulations, both $u_{*}$ and $U$ collapse onto a single curve when plotted against $E_{a}$ (Figs. 10b, 11). As $F_{B}$ strengthens as compared to $F_{C}, u *$ decreases linearly with $E_{a}$. While $U$ also decreases with increasing
$E_{a}$, this modification is not linear in $E_{a}$ due to the quadratic relationship given in (9).

\section{c. Relaminarized regime: $0<E_{a}<1$ and $E_{L}=1$}

For experiments where $E_{a}$ approaches 1 but $E_{L} \approx 1$, the BBL dynamics enter a state that we refer to as a relaminarized stage; the distinction between this state and the arrested state has not previously been documented. The relaminarized stage can be identified when properties are averaged over a time comparable to the inertial period. However, at subinertial time scales, the simulations exhibit strong oscillations in all turbulent properties. Earlier studies have shown similar results (e.g., Umlauf et al. 2015), although these features were not discussed. We begin by summarizing the time-averaged characteristics of this stage, and then provide further details on the near-inertial resonant behavior.

For cases where the buoyancy force is of leading order, the pycnocline does not sharpen noticeably during the evolution of the BML-the ratio of pycnocline stratification to background stratification is roughly 1 (Fig. 7c). Not only does the pycnocline remain weak, but the background stratification also penetrates from the top of the BML downward when $E_{L}$ approaches 1 (Fig. 7c). This restratification is related to the viscous 

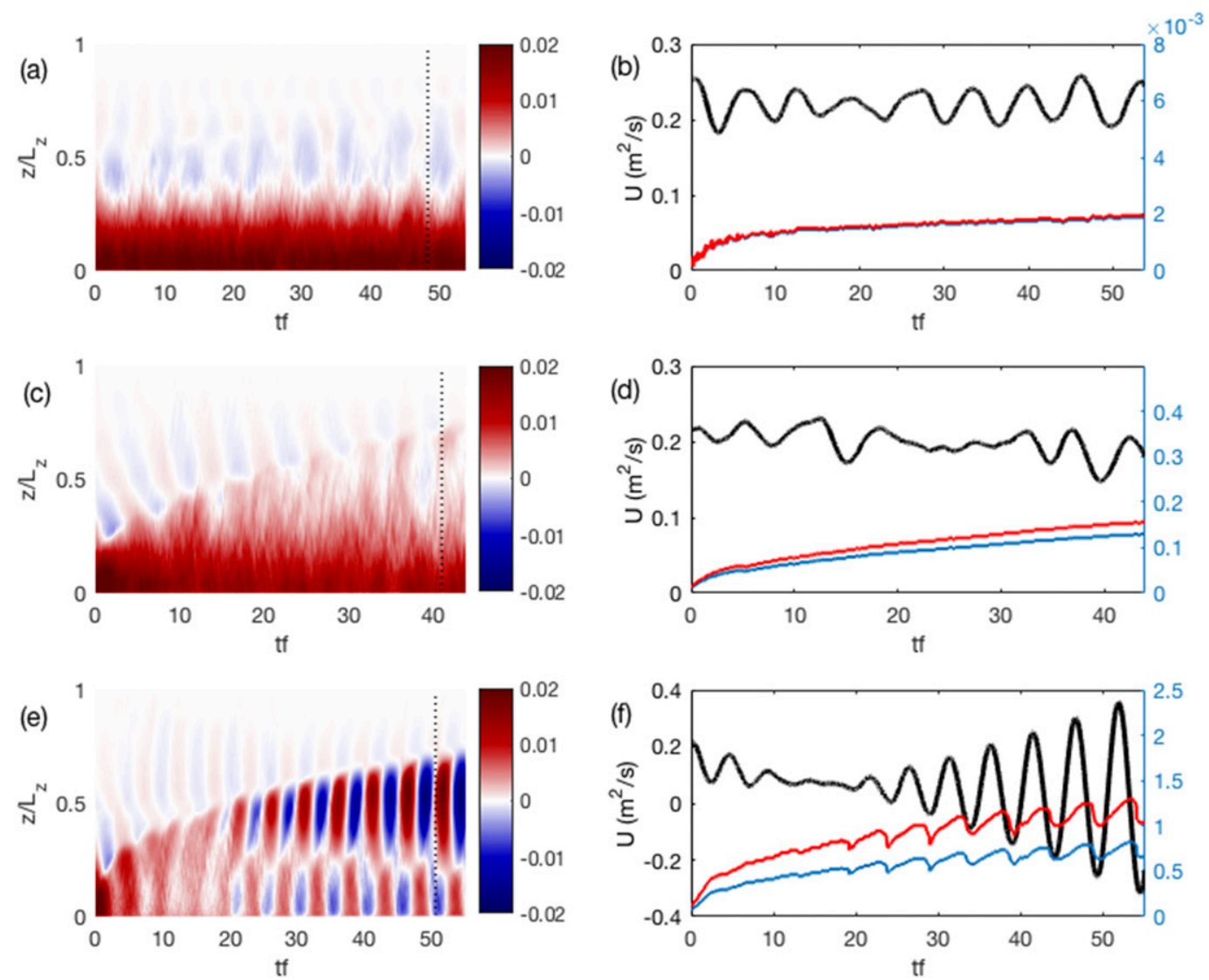

FIG. 9. The evolution of (left) cross-slope velocity $\left(\mathrm{m} \mathrm{s}^{-1}\right)$ and (right) depth-integrated transport $U\left(\mathrm{~m}^{2} \mathrm{~s}^{-1}\right)$ for simulations (a),(b) A, (c),(d) D, and (e),(f) F. The corresponding $E_{a}$ and $E_{L}$ for each simulation are shown in blue and red curves in the transport panels, respectively, with the corresponding axis on the right in blue. The vertical dotted lines in the cross-slope velocity panels represent the centers of the time-averaging windows (of a nearinertial period) used to generate the vertical structures of the velocity variables and other components in the momentum and buoyancy budgets.

slope Obukhov length $L_{s}^{+}$and is discussed further below. The total cross-slope transport arrives at a negligible, but nonzero, value; for example, in experiment $\mathrm{F}$, this occurs after $t f=20$ (Fig. 9f). The friction velocity continues to decrease linearly with $E_{a}$, but remains finite even when $L_{s}^{+}$approaches 100 (Fig. 10a, 12b), as predicted in section $2 \mathrm{~b}$. In experiment $\mathrm{F}$, when $L_{s}^{+}$approaches 100 , the nearbottom velocity $V_{b}$ is smaller than $0.05 \mathrm{~m} \mathrm{~s}^{-1}$, which is half of the along-slope mean flow magnitude $V_{\infty}=0.1 \mathrm{~m} \mathrm{~s}^{-1}$ (Fig. 8b). A reduction in the near-bottom velocity by a factor of 2 results in a reduction of the wall stress by a factor of 4 [(13)] and a reduction in the bottom dissipation rate by a factor of 8 , as compared with the predictions using the far-field mean flow $V_{\infty}$.

From the mean momentum budget [(6)], the predicted arrest height for experiment $\mathrm{F}$ is $H_{a} \approx 50 \mathrm{~m}$. This value is larger than the simulated BML thickness in the relaminarized stage, $\sim 30 \mathrm{~m}$, consistent with $E_{a}<1$. The use of (14), however, requires an estimate of the drag coefficient $C_{d}$. We evaluate $C_{d}=2.2 \times 10^{-3}$ at the beginning of experiment $\mathrm{F}$ before stratification is introduced, using

$$
C_{d}=u_{*}^{2} / V_{b}^{2} .
$$

Plugging in the value of $C_{d}$ and the relaminarization constant $C$ diagnosed earlier, the predicted $H_{L}$ is $31.7 \mathrm{~m}$, which matches the simulated height well. This demonstrates that the BBL relaminarization condition is met before the traditional complete Ekman arrested state.

As experiments $\mathrm{F}$ and $\mathrm{H}$ reach $E_{L} \approx 1$, the boundary layer relaminarizes with negligible turbulent kinetic energy (TKE; e.g., at $t f=50$ in experiment F; Fig. 12a). The value of $L_{s}^{+}$that corresponds to this relaminarization is roughly 100 in both cases, which is the same value reported by Flores and Riley (2011) using the viscous Obukhov length scale $L u * / \nu$ (Fig. 12b). With $C=100$, the predicted friction velocities in the arrested boundary 

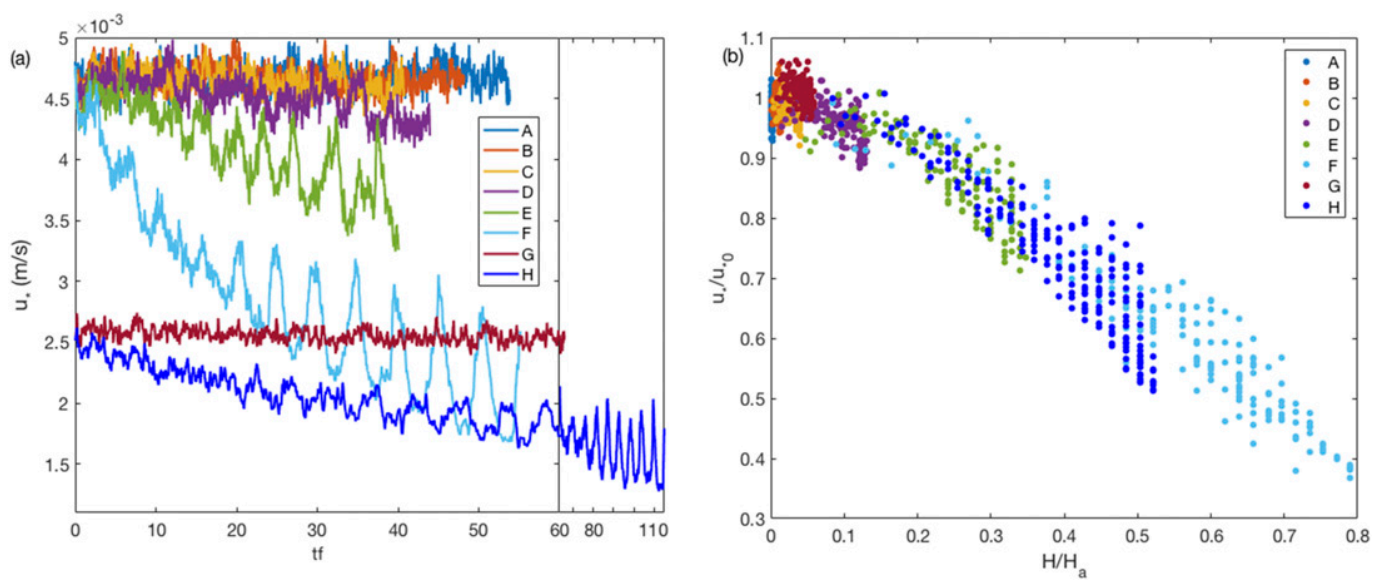

FIG. 10. (a) The evolution of friction velocity $u^{*}\left(\mathrm{~m} \mathrm{~s}^{-1}\right)$ as a function of time. (b) The evolution of friction velocity $u *$, nondimensionalized by the initial friction velocity $u_{*_{0}}$, as a function of $E_{a} \equiv H / H_{a}$. Different colors represent different simulations in Table 1.

layer from (12), using parameters from experiments $\mathrm{F}$ and $\mathrm{H}$, are $u_{*}=1.71 \times 10^{-3}$ and $u_{*}=1.37 \times 10^{-3} \mathrm{~m} \mathrm{~s}^{-1}$, respectively, which agree with the simulated values of $u *$ in Fig. 10a. The arrested wall stress and friction velocity remain finite, as predicted from section $2 \mathrm{~b}$.

Another prominent feature of the large $E_{L}$ regime is the appearance and growth of strong oscillations and resonant behavior. These appear in almost all of the properties discussed above. For instance, both crossslope transport and TKE oscillate, and the amplitude of these oscillations grows with time (Figs. 9f, 12a). The friction velocity oscillates at a near-inertial frequency, but the amplitude does not grow with time. These growing oscillations in cross-slope transport give rise to

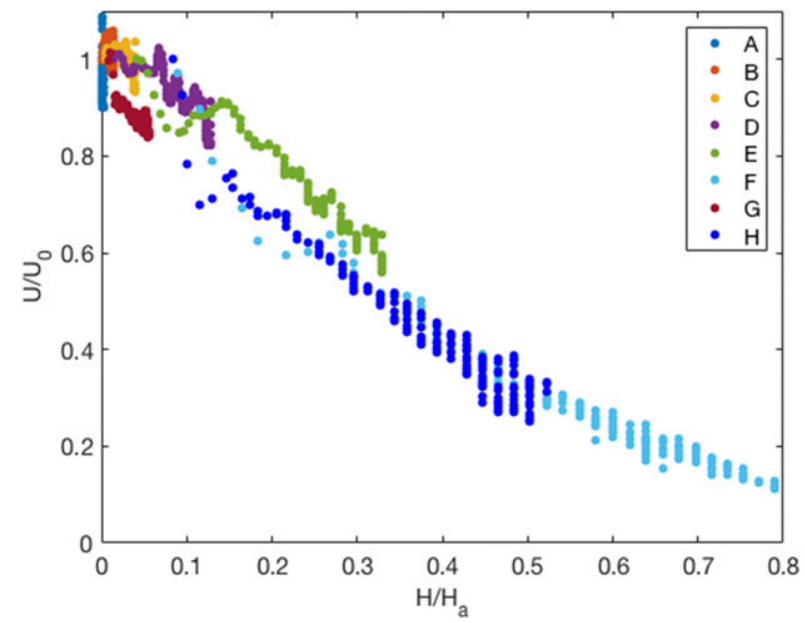

FIG. 11. Plane-averaged cross-slope transport $U$, nondimensionalized by the initial transport $U_{0}$, as a function of $E_{a} \equiv H / H_{a}$. A running mean filter is applied with an averaging window of $5 / f$ to remove the large near-inertial oscillations in $U$. Different colors correspond to the experiments listed in Table 1. bursts in TKE (Figs. 9f, 12a). Even though the crossslope transport averaged over each near-inertial cycle is decaying toward the arrested value, the maximum amplitude of $U$ continues to grow. This indicates an underlying resonant interaction between the stratification and turbulent motions. Analysis of the phase relation among the stratification, TKE, and turbulent momentum flux shows that each time the isopycnals tilt downslope, the stabilizing effect from the stratification vanishes, resulting in a burst of TKE and turbulent momentum flux convergence in the BML. This then advects the isopycnals farther downslope. When the near-inertial oscillation advects the isopycnals upslope, turbulence becomes suppressed at the same time that the stratification strengthens, which results in negligible TKE. The intrinsic frequency can be identified as

$$
\omega=\left(f^{2}+\alpha^{2} N_{\infty}^{2}\right)^{1 / 2}
$$

the inertial frequency is modified by the slope angle and background stratification (BL10). In the relaminarized stage, background turbulence becomes weak, such that all of the key properties that influence the BBL (e.g., thermal wind shear, cross-slope transport, and wall stress) oscillate at the same frequency $\omega$ (Figs. 7c, 9f, 10a), and resonance is likely to occur. In the ocean, resonant behavior may be disrupted or suppressed by temporal variability in the mean flow arising from surface forcing, tides or internal waves, or background dissipation associated with wave breaking.

Although $u *$ decreases as $E_{a}$ increases, leading to a larger viscous length scale $\nu / u *$, the near-bottom log-law layer, in fact, becomes shallower (Fig. 13). The log-law layer disappears when $z^{+}=z u * / \nu$ reaches 150 in the arrested $\mathrm{BBL}$, whereas it remains intact to at least $z^{+}=2000$ in other stages. These values of $z^{+}$correspond 

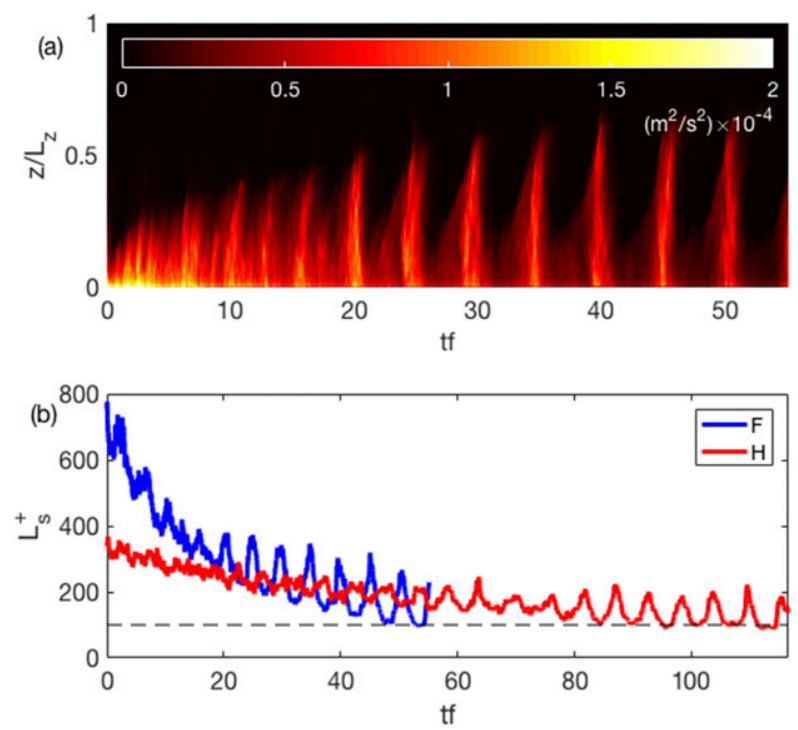

FIG. 12. The evolution of (a) TKE $\left(\mathrm{m}^{2} \mathrm{~s}^{-2}\right)$ for simulation $\mathrm{F}$ and (b) viscous slope Obukhov length $L_{s}^{+}[(11)]$ for simulations F (blue) and $\mathrm{H}$ (red). The dashed line represents $L_{s}^{+}=100$.

to 4.4 and $21.6 \mathrm{~m}$ in dimensional units with the updated viscous length scale. This places constraints on the first grid point in the near-wall modeling when wall models are applied.

\section{d. Ekman arrested regime: $E_{a}=1$ and $E_{L}>1$}

Simulations presented in this study did not achieve steady Ekman arrest because of the long adjustment by molecular diffusion needed to reach this state. This regime transition was not identified in studies that parameterized $\mathrm{BBL}$ turbulence. Also, although the averaged quantities over a near-inertial period (e.g., $U$, $u *$, and TKE) continue to decay slowly, the oscillations appear to grow stronger, especially for $U$ and TKE (Figs. 9, 12). It is unknown if these large oscillations will interrupt the Ekman arrested state. Finally, the fully arrested state has been shown to be susceptible to instabilities (e.g., symmetric instability; Allen and Newberger 1998) that may also generate turbulent motions and drive the BBL away from the arrested state.

\section{e. BBL turbulence}

As discussed in section $2 \mathrm{~b}$, the Monin-Obukhov length scale $L[(7)]$ describes the evolution of turbulent characteristics in the BBL under both stable and unstable conditions. Previous work has shown that for $H / L<0$, the boundary layer is unstable; for $0<H / L<1$, the boundary layer remains neutral; for $1<H / L<10$, the boundary layer is stable; and for $H / L>10$, the boundary layer turbulence becomes intermittent (Holtslag and Nieuwstadt 1986).
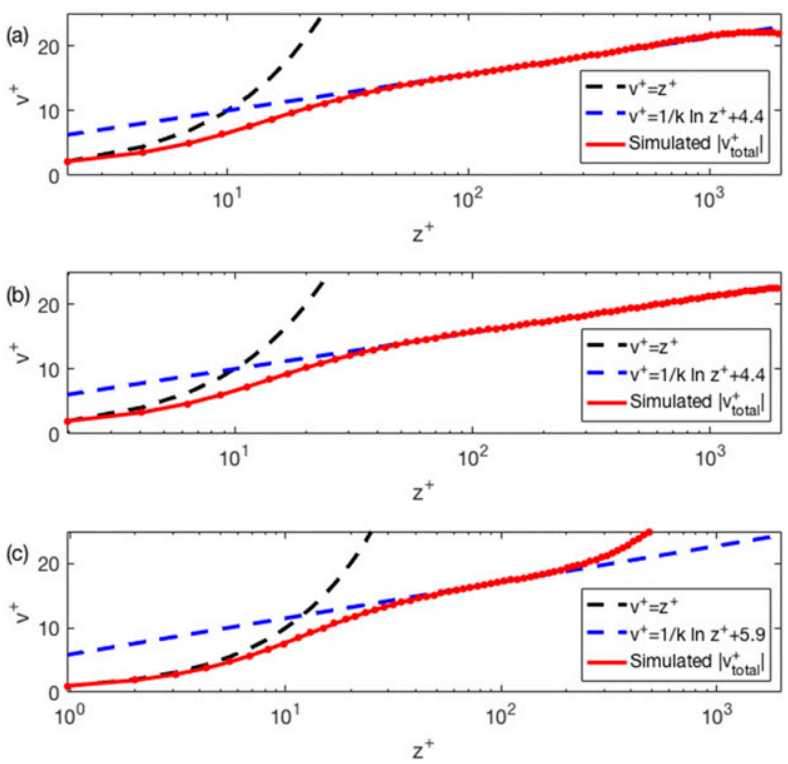

FIG. 13. The magnitude of the nondimensional total along-slope velocity $\left|v_{\text {total }}^{+}\right|=\left(V_{\infty}-v\right) / u_{*}$, as a function of the nondimensional height above the bottom $z^{+}=z u * / \nu$, in simulations (a) A, (b) D, and (c) F. The dashed black and blue curves denote the linear and logarithmic velocity profiles. The red curve is the simulated $\left|v_{\text {total }}^{+}\right|$, with each dot representing a grid point.

In these LES, we find that $E_{L}\left(=H / H_{L}\right)$ is directly related to $H / L_{s}$, where the latter nondimensional parameter is defined using the new slope Obukhov length $L_{s}$ (Fig. 14). The BBL is unstable from the start of the simulation where an upward buoyancy flux is generated by the downslope advection of light fluid (Fig. 15a). The buoyancy flux becomes intermittent later in the experiment with positive pulses only evident in the downslope phase of the growing near-inertial oscillations (Fig. 15b). The oscillations feature periods with a stabilized BBL; the transition occurs near $E_{L} \sim 0.2$ and $H / L_{s} \sim 1$. This is different from the classic Monin-Obukhov scaling since $H / L_{s}$ does not change sign between unstable and stable BBLs. The impact of $H / L_{s}$ on the BBL evolution will be the focus of future studies. We conclude this section by summarizing the various stages in the Ekman arrest process based on nondimensional parameters $\left(E_{a}\right.$ and $\left.E_{L}\right)$, the momentum balance, and the near-bottom velocity magnitude $V_{b}$ (Fig. 5).

\section{Momentum and buoyancy budgets}

We now present plane-averaged budgets of momentum and buoyancy to further illustrate the transition in BBL evolution across the weakly buoyant, buoyant, and relaminarized regimes. The same time average window over a near-inertial period is applied as in section 4 unless otherwise noted. 


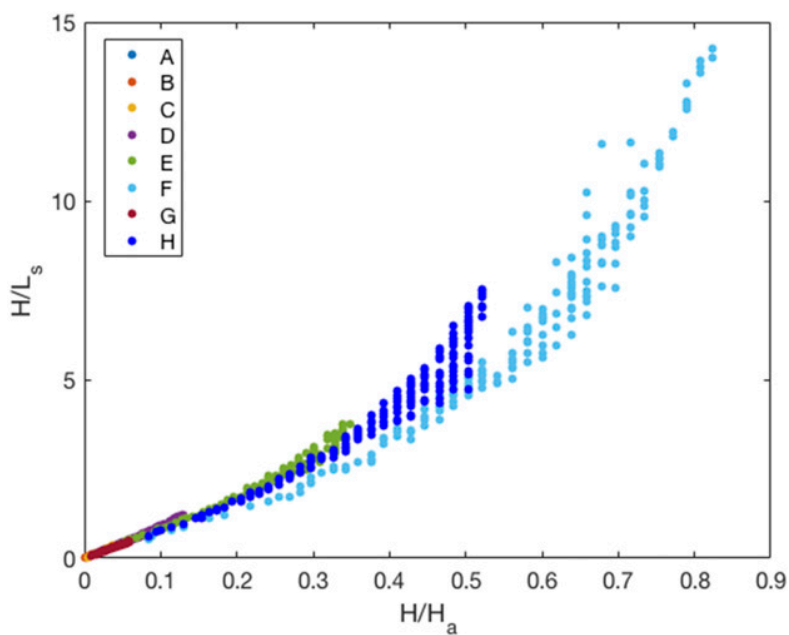

FIG. 14. The relationship between $E_{a} \equiv H / H_{a}$ and $H / L_{s}$. Different colors represent different simulations in Table 1.

\section{a. Momentum budget}

The plane-averaged horizontal momentum equations in the boundary layer can be written as

$$
\frac{\partial\langle u\rangle}{\partial t}-f\langle v\rangle=-b \alpha+\nu \nabla^{2}\langle u\rangle-\frac{\partial\left\langle u^{\prime} w^{\prime}\right\rangle}{\partial z}
$$

$$
\frac{\partial\langle v\rangle}{\partial t}+f\langle u\rangle=\nu \nabla^{2}\langle v\rangle-\frac{\partial\left\langle v^{\prime} w^{\prime}\right\rangle}{\partial z}
$$

where angle brackets denote an average along $x$ and $y$ directions, and $\left\langle u^{\prime} w^{\prime}\right\rangle$ and $\left\langle v^{\prime} w^{\prime}\right\rangle$ are the vertical turbulent fluxes of horizontal momentum, or the Reynolds stresses. The tendency terms in the momentum equations are small, indicating that the simulations are in quasi equilibrium even as the BML grows diffusively, and the viscous terms only become important in the viscous sublayer.

For the cross-slope momentum equation [(36)], three terms may contribute based on the magnitude of $E_{L}$ : the Coriolis force, the buoyancy force, and the Reynolds stress convergence. For small $E_{L}$, the buoyancy force is negligible, and the classic flat-bottom Ekman balance dominates with the Coriolis force balancing the Reynolds stress convergence (Fig. 16a). As $E_{L}$ transitions to $O(0.1)$, the Coriolis, buoyancy, and Reynolds stress convergence terms are all of leading order (Fig. 16b). Since the BML is, by definition, relatively well mixed, the buoyancy force decays roughly linearly with height above bottom (Fig. 16b). Compared to the small $E_{L}$ case, the magnitude and vertical structure of the Reynolds stress convergence
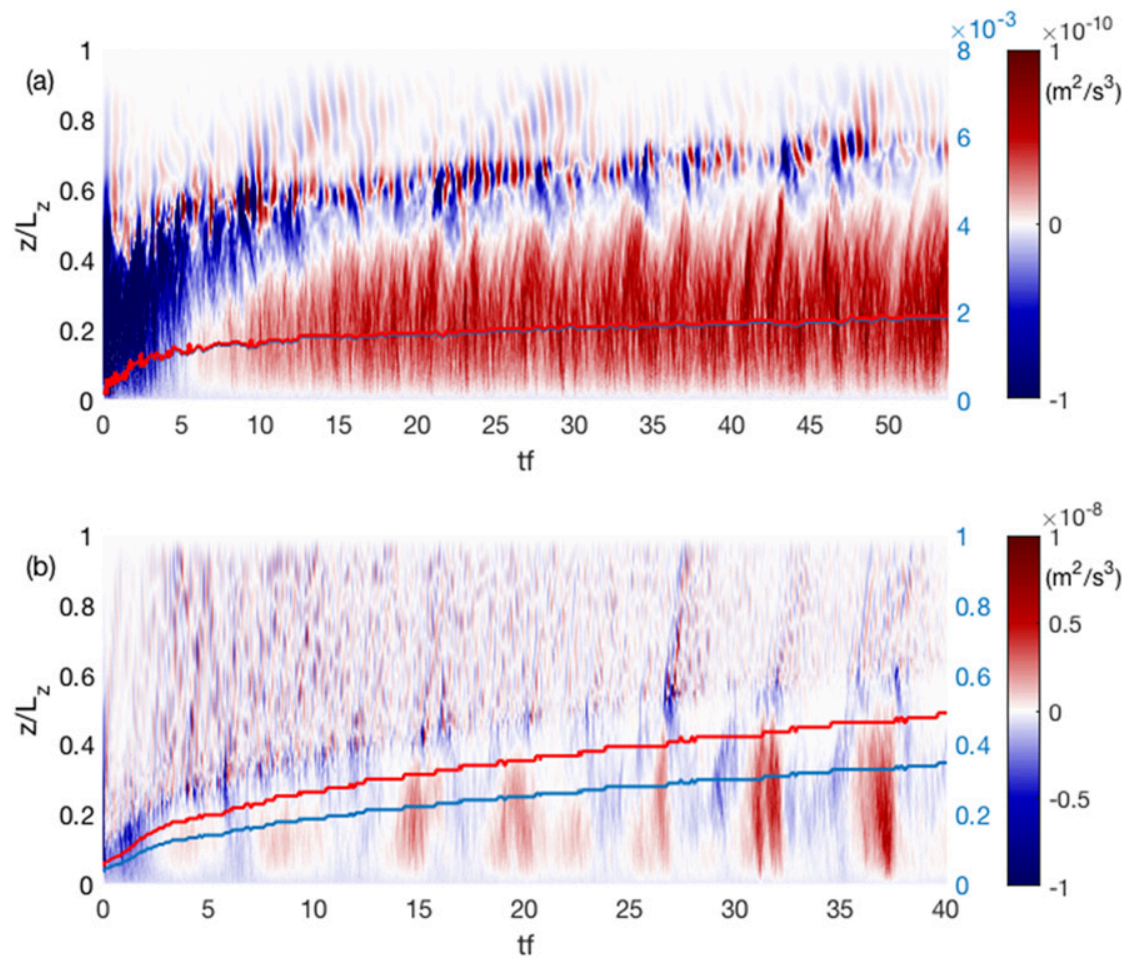

FIG. 15. The evolution of the vertical buoyancy flux as a function of time in simulations (a) A and (b) E. The evolution of the nondimensional parameters $E_{a}$ and $E_{L}$ are given by the blue and red curves, respectively, with the corresponding axis on the right in blue. The BBL is unstable with small $E_{L}$ in simulation $\mathrm{A}$ and transitions from unstable to stable in simulation $\mathrm{E}$ around $t f=5$ when $E_{L}$ exceeds 0.2. 

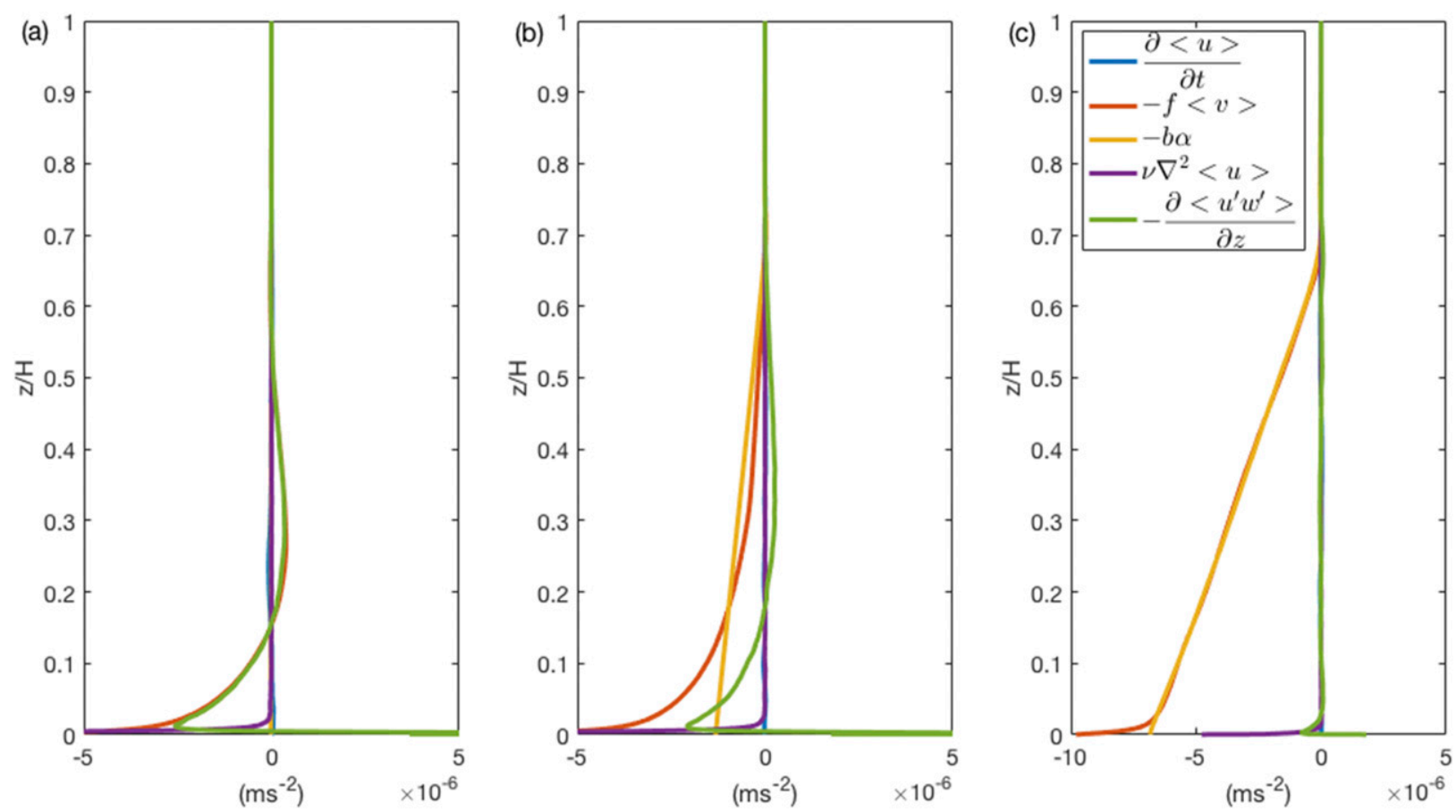

FIG. 16. The momentum balance in the cross-slope direction given in (36) for experiments (a) A, (b) D, and (c) F. The same averaging window is used here as in Fig. 9. The blue curve is the momentum tendency, red curve the Coriolis force, orange curve the buoyancy force, purple curve the molecular friction, and green curve the Reynolds stress convergence.

term remains largely unchanged, but the Coriolis force has a nonnegligible contribution farther away from the bottom. This is consistent with the penetration of the thermal wind shear away from the boundary and farther into the interior. Throughout the BML, $F_{C}$ and $F_{B}$ have the same sign. In this case, the BML remains turbulent, and the cross-slope transport and friction velocity are reduced. The momentum balance changes dramatically as $E_{L}$ approaches 1 and the boundary layer reaches a relaminarized state (Fig. 16c). Now, $F_{C}$ and $F_{B}$ approximately balance in the BML, outside of the thin viscous layer near $z=0$. Turbulence and turbulent fluxes are suppressed in the relaminarized state.

A buoyancy force equivalent to $F_{B}$ does not appear in the along-slope momentum equation [(37)]. Thus, the leading-order balance between Coriolis and Reynolds stress convergence is independent of $E_{L}$ (figure not shown). However, the magnitude of these terms varies significantly both across experiments and during individual experiments. As $E_{L}$ increases, the suppression of turbulence and the reduction in cross-slope Ekman velocity reduces the magnitude of both terms.

\section{b. Buoyancy budget}

The evolution of the plane-averaged buoyancy is described by

$$
\frac{\partial\langle b\rangle}{\partial t}=\langle u\rangle \alpha N_{\infty}^{2}+\kappa \nabla^{2}\langle b\rangle-\frac{\partial\left\langle w^{\prime} b^{\prime}\right\rangle}{\partial z},
$$

where $\left\langle w^{\prime} b^{\prime}\right\rangle$ is the plane-averaged vertical turbulent buoyancy flux. Outside of the viscous sublayer, all terms contribute to the buoyancy budget other than the molecular diffusion term. The cross-slope buoyancy advection occurs mainly in the Ekman layer, which is thinner than the BML (Figs. 17a,b). For these downslope favorable conditions, cross-slope advection generates a local tendency to increase buoyancy. The vertical turbulent buoyancy flux diverges in the lower part of the BBL, opposing the cross-slope advection. However, the turbulent buoyancy flux converges in the upper part of the BBL and, without a contribution from the cross-slope advection, produces a positive buoyancy tendency. Finally, there is a narrow region of divergence of the turbulent buoyancy flux in the pycnocline.

Within a single experiment, the magnitude of buoyancy advection decreases as $E_{L}$ increases, although the advection also penetrates deeper into the interior. However, the buoyancy advection term also depends on the background cross-slope buoyancy gradient $M_{\infty}^{2}=-\alpha N_{\infty}^{2}$, which is related to the initial Bu. Thus, from experiments $\mathrm{A}$ to $\mathrm{D}$, the magnitude of the buoyancy advection terms become larger (Figs. 17a,b). When relaminarization occurs in the boundary layer, 

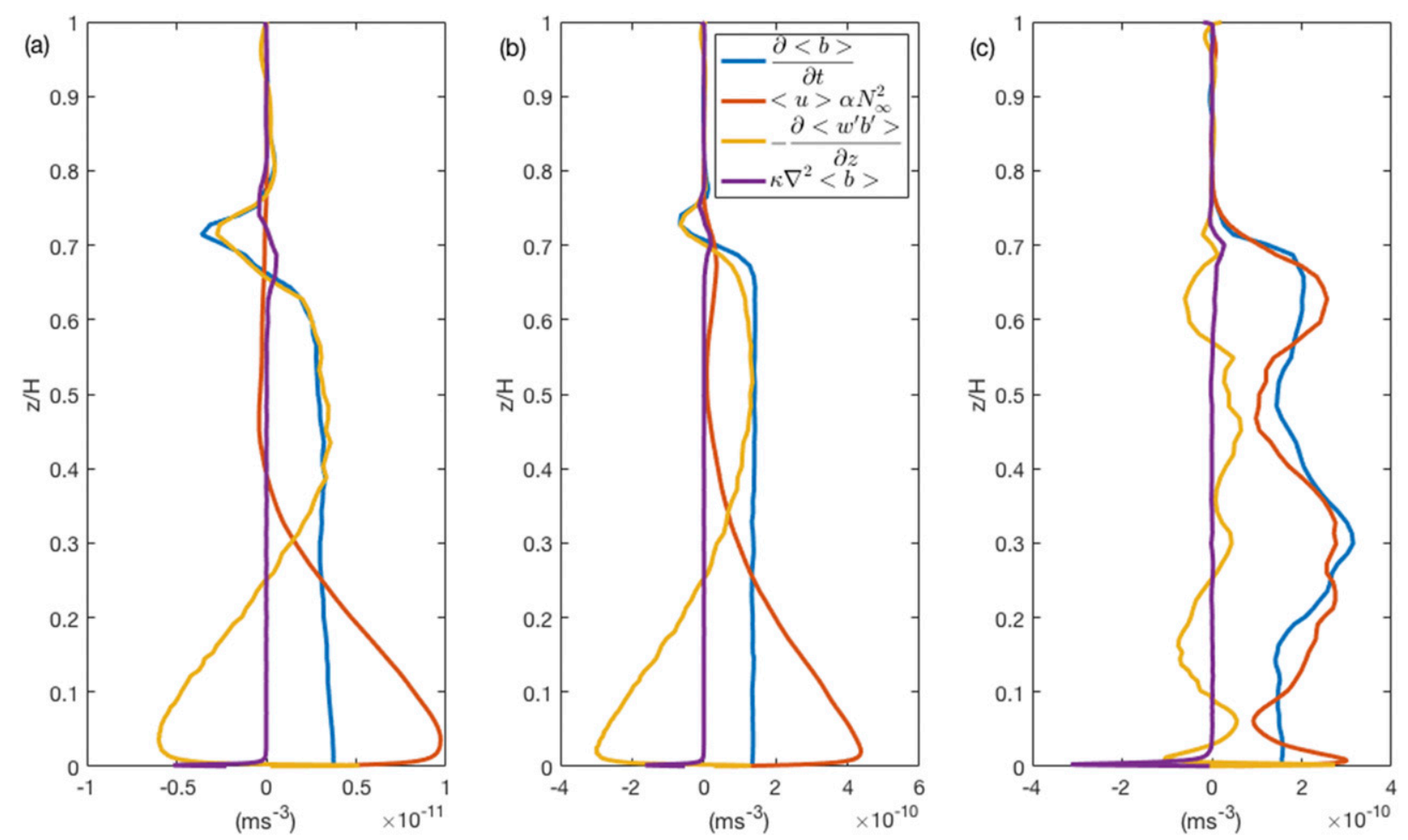

FIG. 17. The buoyancy budget given in (38) for experiments (a) A, (b) D, and (c) F. The same averaging window is used here as in Fig. 9. The blue curve is the buoyancy tendency, red curve the cross-slope buoyancy advection, orange curve the turbulent diffusion, and purple curve the molecular diffusion.

the cross-slope velocity and total cross-slope buoyancy advection are significantly reduced, although they remain finite (Figs. 8a, 17c). As $E_{L}$ approaches 1 , the turbulent buoyancy flux convergence becomes negligible in the buoyancy budget due to the suppression of turbulence.

\section{Discussion and conclusions}

The bulk structure of a stratified oceanic BBL over a smooth slope is explored using both scaling analyses and LES. The key conclusions include the following:

1) We provide expressions that predict the height of the bottom mixed layer (BML) $H$ in a state of Ekman arrest based on the momentum budget $H_{a} \approx f V_{\infty} /\left(\alpha N_{\infty}^{2}\right)$ (see also Trowbridge and Lentz $1991)$ and on the relaminarization condition

$$
H_{L}=\frac{f V_{\infty}}{\alpha N_{\infty}^{2}}-\left[\frac{C k \nu f}{\alpha N_{\infty}^{2} C_{d}\left(1+\mathrm{Bu}^{2}\right)}\right]^{1 / 2} .
$$

We find that $H_{L}$ is always less than $H_{a}$. Two nondimensional parameters, $E_{a}=H / H_{a}[(32)]$ and $E_{L}=H / H_{L}[(33)]$, can be used to determine the sequential stages of the BBL as it approaches full Ekman arrest.

2) We present a new length scale, the slope Obukhov length $L_{s}$, which characterizes the relative importance of turbulence production and cross-slope buoyancy advection [(10)]. Its nondimensional form, the viscous slope Obukhov length $L_{s}^{+}$, can be used to predict the relaminarization condition for the turbulent $\operatorname{BBL}\left(L_{s}^{+} \approx 100\right)$.

3) We predict the wall stress and friction velocity [(12)] when the BBL becomes laminar and the turbulence is suppressed. This can be used to estimate the integrated BBL energy dissipation rate at the relaminarized state.

4) We argue that the complete Ekman arrested state is unlikely to be observed in the real ocean because (i) $H_{a}$ and $H_{L}$ are expected to be large, based on typical deep ocean parameters, which inevitably leads to a long adjustment time scale; (ii) the BBL relaminarization is always achieved before the steady arrested state, and the subsequent molecular adjustment is prone to external perturbations; and (iii) in the rare event of full Ekman arrest, the steady arrested BBL is unstable to symmetric instability (see Allen and Newberger 1998). 
5) We show that the nondimensional parameter $E_{a}$ describes the evolution of the cross-slope transport and wall stress across different regimes in a suite of simulations that vary several parameters, including the slope angle $\alpha$, the background vertical stratification $N_{\infty}^{2}$, and the mean flow magnitude $V_{\infty}$. The relaminarization stage is determined from $E_{L}$. The parameters $E_{a}$ and $E_{L}$ are closely related to the BBL turbulence through the classic Monin-Obukhov similarity theory $\left(H / L_{s}\right)$, and this framework is used to analyze changes in the momentum and buoyancy budgets across different stages toward the arrested state. The potential vorticity evolution will be discussed in a future study.

As $E_{L}$ increases, the BML differs from the flat-bottom case in the following ways: (i) the pycnocline at the top of the BML weakens; (ii) the cross-slope velocity penetrates deeper due to the thermal wind shear near the bottom; and (iii) the velocity shear near the wall, and thus the wall stress, weakens, resulting in a decay of the friction velocity, cross-slope transport, and Ekman veering angle near the bottom. When the BBL relaminarizes, the mean velocity departs from the log-law closer to the bottom.

These results suggest that the interaction between stratification and sloping topography could reduce the contribution of bottom friction to the dissipation of kinetic energy in the ocean. Global quantification of the bottom dissipation rate, using either observations from deep ocean current meters or from numerical models (that typically apply uniform drag coefficients), has not accounted for the modification of near-bottom flows due to the presence of stratification and topographic slopes (Wunsch and Ferrari 2004; Sen et al. 2008; Arbic et al. 2009; Wright et al. 2013). Additionally, recent work has suggested that the ocean's abyssal circulation may be influenced by the thermal wind shear associated with tilting isopycnals at the seafloor (Callies and Ferrari 2018). However, this work typically assumes that the global BBL is largely in the Ekman arrested state. Determining the spatial distribution of $E_{a}$ and $E_{L}$, which can be calculated from observable ocean properties, could shed additional light on the BBL's influence over global dissipation rates and the abyssal circulation.

The BBL over topographic slopes has recently been highlighted as the key region where dense waters can be transformed to lighter density classes to close the overturning circulation (De Lavergne et al. 2016; Ferrari et al. 2016; De Lavergne et al. 2017). Water must also be exchanged between the ocean interior and the boundary layer in order to maintain stratification and sustain this water mass modification. Earlier studies have not accounted for dynamics that will affect mixing rates and BBL-interior exchange. The Ekman arrest process, for instance, could act as a barrier for such exchange via mass flux out of and into the BBL due to mass convergence/divergence when strong near-bottom mean flows or (sub)mesoscale eddies are present. Finally, Ekman arrest characteristics may be sensitive to along-isobath variations that are not considered in this study (Brink 2012). Other factors, such as the level of background turbulence or temporal variability associated with tidal fluctuations in the abyssal ocean, need to be addressed in future studies to estimate the extent to which Ekman arrest is achieved in the ocean.

Acknowledgments. We thank two anonymous reviewers as well as Georgy Manucharyan for helpful comments that improved this manuscript. We gratefully acknowledge support from NSF Awards OPP-1246460 and OPP-1644172.

\section{REFERENCES}

Allen, J., and P. Newberger, 1998: On symmetric instabilities in oceanic bottom boundary layers. J. Phys. Oceanogr., 28, 1131-1151, https://doi.org/10.1175/1520-0485(1998)028<1131: OSIIOB $>2.0 . \mathrm{CO} ; 2$.

Arbic, B. K., and Coauthors, 2009: Estimates of bottom flows and bottom boundary layer dissipation of the oceanic general circulation from global high-resolution models. J. Geophys. Res., 114, C02024, https://doi.org/10.1029/2008JC005072.

Armi, L., 1978: Some evidence for boundary mixing in the deep ocean. J. Geophys. Res., 83, 1971-1979, https://doi.org/10.1029/ JC083iC04p01971.

Benthuysen, J. A., and L. N. Thomas, 2013: Nonlinear stratified spindown over a slope. J. Fluid Mech., 726, 371-403, https:// doi.org/10.1017/jfm.2013.231

Bewley, T. R., 2008: Numerical Renaissance: Simulation, Optimization, and Control. Renaissance Press, $801 \mathrm{pp}$.

Brink, K. H., 2012: Buoyancy arrest and shelf-ocean exchange. J. Phys. Oceanogr., 42, 644-658, https://doi.org/10.1175/ JPO-D-11-0143.1.

— , and S. J. Lentz, 2010: Buoyancy arrest and bottom Ekman transport. Part I: Steady flow. J. Phys. Oceanogr., 40, 621-635, https://doi.org/10.1175/2009JPO4266.1.

Callies, J., and R. Ferrari, 2018: Dynamics of an abyssal circulation driven by bottom-intensified mixing on slopes. J. Phys. Oceanogr., 48, 1257-1282, https://doi.org/10.1175/ JPO-D-17-0125.1.

Coleman, G. N., J. Ferziger, and P. Spalart, 1990: A numerical study of the turbulent Ekman layer. J. Fluid Mech., 213, 313 348, https://doi.org/10.1017/S0022112090002348.

Deardorff, J. W., G. E. Willis, and D. K. Lilly, 1969: Laboratory investigation of non-steady penetrative convection. J. Fluid Mech., 35, 7-31, https://doi.org/10.1017/S0022112069000942.

De Lavergne, C., G. Madec, J. Le Sommer, A. G. Nurser, and A. C. Naveira Garabato, 2016: On the consumption of Antarctic Bottom Water in the abyssal ocean. J. Phys. Oceanogr., 46 635-661, https://doi.org/10.1175/JPO-D-14-0201.1. 
- - - F. Roquet, R. Holmes, and T. McDougall, 2017: Abyssal ocean overturning shaped by seafloor distribution. Nature, 551, 181-186, https://doi.org/10.1038/nature24472.

Deusebio, E., G. Brethouwer, P. Schlatter, and E. Lindborg, 2014: A numerical study of the unstratified and stratified Ekman layer. J. Fluid Mech., 755, 672-704, https://doi.org/ 10.1017/jfm.2014.318.

Ferrari, R., A. Mashayek, T. J. McDougall, M. Nikurashin, and J.-M. Campin, 2016: Turning ocean mixing upside down. J. Phys. Oceanogr., 46, 2239-2261, https://doi.org/10.1175/ JPO-D-15-0244.1.

Flores, O., and J. Riley, 2011: Analysis of turbulence collapse in the stably stratified surface layer using direct numerical simulation. Bound.-Layer Meteor., 139, 241-259, https:// doi.org/10.1007/s10546-011-9588-2.

Gula, J., M. J. Molemaker, and J. C. McWilliams, 2016: Topographic generation of submesoscale centrifugal instability and energy dissipation. Nat. Commun., 7, 12811, https://doi.org/ 10.1038/ncomms12811.

Holtslag, A., and F. Nieuwstadt, 1986: Scaling the atmospheric boundary layer. Bound.-Layer Meteor., 36, 201-209, https:// doi.org/10.1007/BF00117468.

MacCready, P., and P. B. Rhines, 1991: Buoyant inhibition of Ekman transport on a slope and its effect on stratified spinup. J. Fluid Mech., 223, 631-661, https://doi.org/10.1017/ S0022112091001581.

Manucharyan, G. E., and C. Caulfield, 2015: Entrainment and mixed layer dynamics of a surface-stress-driven stratified fluid. J. Fluid Mech., 765, 653-667, https://doi.org/10.1017/ jfm.2015.5.

Munk, W. H., 1966: Abyssal recipes. Deep-Sea Res. Oceanogr. Abstr., 13, 707-730, https://doi.org/10.1016/0011-7471(66)90602-4.

Nikurashin, M., and R. Ferrari, 2011: Global energy conversion rate from geostrophic flows into internal lee waves in the deep ocean. Geophys. Res. Lett., 38, L08610, https://doi.org/10.1029/ 2011GL046576.

Phillips, O., 1970: On flows induced by diffusion in a stably stratified fluid. Deep-Sea Res. Oceanogr. Abstr., 17, 435-443, https:// doi.org/10.1016/0011-7471(70)90058-6.

Pope, S. B., 2001: Turbulent Flows. Cambridge University Press, $771 \mathrm{pp}$.

Ruan, X., and A. F. Thompson, 2016: Bottom boundary potential vorticity injection from an oscillating flow: A PV pump. J. Phys. Oceanogr., 46, 3509-3526, https://doi.org/10.1175/ JPO-D-15-0222.1.

M. M. Flexas, and J. Sprintall, 2017: Contribution of topographically generated submesoscale turbulence to Southern Ocean overturning. Nat. Geosci., 10, 840-845, https://doi.org/10.1038/ngeo3053.

Sagaut, P., 2006: Large Eddy Simulation for Incompressible Flows: An Introduction. Springer, $558 \mathrm{pp}$.
Scott, R. B., and Y. Xu, 2009: An update on the wind power input to the surface geostrophic flow of the World Ocean. Deep-Sea Res. I, 56, 295-304, https://doi.org/10.1016/j.dsr.2008.09.010.

Sen, A., R. B. Scott, and B. K. Arbic, 2008: Global energy dissipation rate of deep-ocean low-frequency flows by quadratic bottom boundary layer drag: Computations from currentmeter data. Geophys. Res. Lett., 35, L09606, https://doi.org/ 10.1029/2008GL033407.

Shingai, K., and H. Kawamura, 2002: Direct numerical simulation of turbulent heat transfer in the stably stratified Ekman layer. Therm. Sci. Eng., 10, 1-9.

Taylor, J. R., 2008: Numerical simulations of the stratified oceanic bottom boundary layer. Ph.D. dissertation, University of California, San Diego, 230 pp., https://escholarship.org/uc/item/5s30n2ts. , and S. Sarkar, 2008: Stratification effects in a bottom Ekman layer. J. Phys. Oceanogr., 38, 2535-2555, https://doi.org/ 10.1175/2008JPO3942.1.

_ , and R. Ferrari, 2010: Buoyancy and wind-driven convection at mixed layer density fronts. J. Phys. Oceanogr., 40, 12221242, https://doi.org/10.1175/2010JPO4365.1.

Thomas, L. N., and P. B. Rhines, 2002: Nonlinear stratified spinup. J. Fluid Mech., 473, 211-244, https://doi.org/10.1017/ S0022112002002367.

Thorpe, S., 1987: Current and temperature variability on the continental slope. Philos. Trans. Roy. Soc. London, 323A, 471517, https://doi.org/10.1098/rsta.1987.0100.

Trowbridge, J., and S. Lentz, 1991: Asymmetric behavior of an oceanic boundary layer above a sloping bottom. J. Phys. Oceanogr., 21, 1171-1185, https://doi.org/10.1175/ 1520-0485(1991)021<1171:ABOAOB > 2.0.CO;2.

—_ and —_, 1998: Dynamics of the bottom boundary layer on the Northern California shelf. J. Phys. Oceanogr., 28, 2075-2093, https://doi.org/10.1175/1520-0485(1998)028<2075: DOTBBL $>2.0 . C O ; 2$

Umlauf, L., W. D. Smyth, and J. N. Moum, 2015: Energetics of bottom Ekman layers during buoyancy arrest. J. Phys. Oceanogr., 45, 3099-3117, https://doi.org/10.1175/JPO-D-15-0041.1.

Weatherly, G. L., and P. J. Martin, 1978: On the structure and dynamics of the oceanic bottom boundary layer. J. Phys. Oceanogr., 8, 557-570, https://doi.org/10.1175/1520-0485 (1978)008<0557:OTSADO >2.0.CO;2.

Wright, C. J., R. B. Scott, D. Furnival, P. Ailliot, and F. Vermet, 2013: Global observations of ocean-bottom subinertial current dissipation. J. Phys. Oceanogr., 43, 402-417, https://doi.org/ 10.1175/JPO-D-12-082.1.

Wunsch, C., 1970: On oceanic boundary mixing. Deep-Sea Res. Oceanogr. Abstr., 17, 293-301, https://doi.org/10.1016/ 0011-7471(70)90022-7.

, and R. Ferrari, 2004: Vertical mixing, energy, and the general circulation of the oceans. Annu. Rev. Fluid Mech., 36, 281-314, https://doi.org/10.1146/annurev.fluid.36.050802.122121. 